LTH 916

July 2011

DESY 11-108

SFB/CPP-11-33

LPN11-34

\title{
On the Next-to-Next-to-Leading Order Evolution of Flavour-Singlet Fragmentation Functions
}

\author{
A.A. Almasy ${ }^{a}, \mathrm{~S} . \mathrm{Moch}^{b}$ and A. $\operatorname{Vogt}^{a}$ \\ ${ }^{a}$ Department of Mathematical Sciences, University of Liverpool \\ Liverpool L69 3BX, United Kingdom \\ ${ }^{b}$ Deutsches Elektronensynchrotron DESY \\ Platanenallee 6, D-15738 Zeuthen, Germany
}

\begin{abstract}
We present the third-order contributions to the quark-gluon and gluon-quark timelike splitting functions for the evolution of fragmentation functions in perturbative QCD. These quantities have been derived by studying physical evolution kernels for photon- and Higgs-exchange structure functions in deep-inelastic scattering and their counterparts in semi-inclusive annihilation, together with constraints from the momentum sum rule and the supersymmetric limit. For this purpose we have also calculated the second-order coefficient functions for one-hadron inclusive Higgs decay in the heavy-top limit. A numerically tolerable uncertainty remains for the quark-gluon splitting function, which does not affect the endpoint logarithms for small and large momentum fractions. We briefly discuss these limits and illustrate the numerical impact of the third-order corrections. Compact and accurate parametrizations are provided for all third-order timelike splitting functions.
\end{abstract}


In this article we address the scale dependence (evolution) of the parton fragmentation distributions (functions) $D_{f}^{h}\left(x, Q^{2}\right)$, see Ref. [1] for an introductory overview, at the next-to-next-to-leading order (NNLO) in massless perturbative QCD. Here $x$ represents the fractional momentum of the final-state parton $f$ transferred to the outgoing hadron $h$; and $Q^{2}$ is a (timelike) hard scale, for instance the squared momentum of the virtual photon or $Z$-boson in semi-inclusive electron-positron annihilation (SIA), $e^{+} e^{-} \rightarrow \gamma, Z \rightarrow h+X$, where $X$ stands for all accessible hadronic states.

The evolution of the fragmentation distributions is given by

$$
\frac{d}{d \ln Q^{2}} D_{a}^{h}\left(x, Q^{2}\right)=\int_{x}^{1} \frac{d z}{z} P_{b a}^{T}\left(z, \alpha_{\mathrm{s}}\left(Q^{2}\right)\right) D_{b}^{h}\left(\frac{x}{z}, Q^{2}\right),
$$

where the summation over $b=q_{i}, \bar{q}_{i}, g$ for $i=1, \ldots, n_{f}$ is understood, and $n_{f}$ denotes the number of effectively massless quark flavours. Unlike the functions $D_{f}^{h}\left(x, Q^{2}\right)$, the 'timelike' splitting functions $P_{b a}^{T}$ can be expanded in powers of the strong coupling $\alpha_{\mathrm{s}}$,

$$
P_{b a}^{T}\left(x, \alpha_{\mathrm{s}}\left(Q^{2}\right)\right)=a_{\mathrm{s}} P_{b a}^{(0) T}(x)+a_{\mathrm{s}}^{2} P_{b a}^{(1) T}(x)+a_{\mathrm{s}}^{3} P_{b a}^{(2) T}(x)+\ldots
$$

We normalize the expansion parameter as $a_{\mathrm{s}}=\alpha_{\mathrm{s}} /(4 \pi)$ and use, without loss of information, the standard $\overline{\mathrm{MS}}$ scheme with the choice $\mu_{r}^{2}=\mu_{f}^{2}=Q^{2}$ for the renormalization and fragmentation (final-state mass factorization) scale. The system (1) of $\left(2 n_{f}+1\right) \times\left(2 n_{f}+1\right)$ coupled equations can be decomposed into $2 n_{f}+1$ scalar flavour non-singlet equations and the $2 \times 2$ flavour-singlet system

$$
\frac{d}{d \ln Q^{2}}\left(\begin{array}{c}
D_{\mathrm{q}} \\
D_{\mathrm{g}}
\end{array}\right)=\left(\begin{array}{cc}
P_{\mathrm{qq}}^{T} & P_{\mathrm{gq}}^{T} \\
P_{\mathrm{qg}}^{T} & P_{\mathrm{gg}}^{T}
\end{array}\right) \otimes\left(\begin{array}{c}
D_{\mathrm{q}} \\
D_{\mathrm{g}}
\end{array}\right) \quad \text { with } \quad D_{\mathrm{q}}=\sum_{i=1}^{n_{f}}\left(D_{q_{i}}+D_{\bar{q}_{i}}\right) .
$$

Here $\otimes$ abbreviates the convolution in Eq. (1), and we have suppressed all functional dependences.

The leading-order (LO) splitting functions $P^{(0) T}(x)$ [2] are identical to their 'spacelike' counterparts [3] for the evolution of the initial-state parton distributions (where the matrix in Eq. (3) is transposed), a fact often referred to as the Gribov-Lipatov relation [4]. The next-to-leading order contributions $P^{(1) T}$ were derived by several groups about thirty years ago [5-9]. Unlike the spacelike case $[10,11]$, where the calculations can be performed via forward scattering amplitudes, the NNLO corrections $P^{(2) T}$ have eluded a direct calculation in terms of Feynman diagrams so far.

The (three) non-singlet quantities $P_{\mathrm{ns}}^{(2) T}$ and the diagonal entries in Eq. (3) have been determined by two of us a couple of years ago $[12,13]$ via analytic continuations (see below) of the unfactorized partonic structure functions from the spacelike (deep-inelastic scattering, DIS) to the timelike SIA case, supplemented by complementary considerations based on Ref. [14]. Only the second Mellin moments of $P_{\mathrm{gq}}^{(2) T}(x)$ and $P_{\mathrm{qg}}^{(2) T}(x)$ are fully known at this point [13], since these quantities are fixed by the momentum sum rule and the diagonal entries.

Beyond the leading order, there is no direct relation between the spacelike splitting functions, or their $x \rightarrow 1 / x$ analytic continuations $(A C)$, and their timelike counterparts in the $\overline{\mathrm{MS}}$ scheme. However such a relation exists for spacelike and timelike physical evolution kernels $K\left(x, \alpha_{\mathrm{s}}\right)$ for photon-exchange DIS and SIA structure functions at NLO [15], see also Ref. [16]. Defining the expansion coefficients $K^{(n)}$ as in Eq. (2), it can schematically be written as

$$
A C\left[K^{(n) S}(x)\right]=K^{(n) T}(x) \text { for } n=0,1 .
$$


In order to access all four splitting functions, we study the physical evolution kernels for the system $F_{1}$ and $F_{\phi}$ of flavour-singlet DIS structure function. The former quantity is chosen, instead of $F_{2}$ in Ref. [17], since it directly corresponds to the transverse fragmentation function $F_{T}$ in SIA. The NNLO coefficient functions for these observables are known from Refs. $[18,19]$, see also Refs. [20,21]. $F_{\phi}$ is the structure function for DIS by the exchange of a scalar $\phi$ coupling directly only to gluons via a term $\phi G^{\mu \nu} G_{\mu v}$ in the Lagrangian (such as the Higgs boson in the limit of a five massless flavours and a very heavy top quark), where $G^{\mu \nu}$ denotes the gluon field strength tensor. The NNLO coefficient functions for the structure function $F_{\phi}$ have been calculated in Refs. [17,22], while those for the corresponding fragmentation function $F_{\phi}^{T}$ are presented in Appendix A.

The spacelike physical kernels have been discussed, for the system $\left(F_{2}, F_{\phi}\right)$, in detail in Ref. [17]. The timelike case is completely analogous up to a transposition of the matrices:

with

$$
\left.\frac{d}{d \ln Q^{2}} F^{T}=K^{T} \otimes F^{T}=\left\{\left(\beta \frac{d C^{T}}{d a_{\mathrm{s}}}+C^{T} \otimes P^{T}\right) \otimes\left(C^{T}\right)^{-1}\right)\right\} \otimes F^{T}
$$

$$
F^{T}=\left(\begin{array}{c}
F_{T} \\
F_{\phi}^{T}
\end{array}\right), \quad K^{T}=\sum_{n=0} a_{\mathrm{s}}^{n+1}\left(\begin{array}{cc}
K_{\mathrm{TT}}^{(n)} & K_{\mathrm{T} \phi}^{(n)} \\
K_{\phi \mathrm{T}}^{(n)} & K_{\phi \phi}^{(n) T}
\end{array}\right), \quad C^{T}=\sum_{n=0} a_{\mathrm{s}}^{n}\left(\begin{array}{cc}
C_{T, \mathrm{q}}^{(n)} & C_{\phi, \mathrm{q}}^{(n) T} \\
C_{T, \mathrm{~g}}^{(n)} & C_{\phi, \mathrm{g}}^{(n) T}
\end{array}\right)
$$

where $c_{T, \mathrm{q}}^{(0)}=C_{\phi, \mathrm{g}}^{(0) T}=\delta(1-x)$ and $c_{T, \mathrm{~g}}^{(0)}=C_{\phi, \mathrm{q}}^{(0) T}=0$. We have skipped the superscript ' $T$ ' where it is not needed for uniqueness in the present context. It should be noted that the normalization of $c_{T, \mathrm{~g}}^{(n)}$ in Eq. (6) differs by a factor of $1 / 2$ from that in Refs. [19,21]. Finally $\beta$ in Eq. (5) is the standard beta function of QCD, $\beta=-\beta_{0} a_{\mathrm{s}}^{2}+\ldots$ with $\beta_{0}=11 / 3 C_{A}-2 / 3 n_{f}$ and $C_{A}=N_{\text {colours }}=3$.

For the off-diagonal entries the analytic continuation involves, besides $x \rightarrow 1 / x$ and the multiplication by a factor $x$ due to the phase space of the detected parton in the SIA case [19], a sign factor and a ratio of colour factors, leading to (with $C_{F}=4 / 3$ in QCD)

$$
A C\left[K_{2 \phi}^{(n)}(x)\right]=-\frac{C_{F}}{n_{f}} x K_{2 \phi}^{(n)}(1 / x), \quad A C\left[K_{\phi 2}^{(n)}(x)\right]=-\frac{n_{f}}{C_{F}} x K_{\phi 2}^{(n)}(1 / x) .
$$

The critical part of the analytic continuation is that of powers of $\ln (1-x)$, which is given by

$$
\ln (1-x) \stackrel{A C_{\kappa}}{\longrightarrow} \ln (1-x)-\ln x+\kappa i \pi \quad \text { with } \quad \kappa=0 \text { or } 1 .
$$

For $\kappa=1$ the real part is taken in the end. It is not clear at all that beyond NLO Eq. (8) is applicable, in either form, to quantities such as physical evolution kernels instead of to (classes of) Feynman diagrams, see the discussions in Refs. $[5,8,16]$.

The NLO physical kernels for $\left(F_{1}, F_{\phi}\right)$ and $\left(F_{T}, F_{\phi}^{T}\right)$ fulfil Eq. (4) for both $\kappa=0$ and $\kappa=1$ in Eq. (8). However, for the NNLO diagonal entries we find (restricting ourselves to $x<1$ )

$$
A C_{0}\left[K_{11}^{(2)}(x)\right]-K_{T T}^{(2)}(x)=24 \zeta_{2} \beta_{0} C_{F}^{2} \frac{1+x^{2}}{1-x} \ln x=12 \zeta_{2} \beta_{0} C_{F} P_{\mathrm{qq}}^{(0)}(x) \ln x,
$$

and a completely analogous relation with $P_{\mathrm{gg}}^{(0)}=K_{\phi \phi}^{(0)}$ on the right-hand-side for $K_{\phi \phi}^{(2)}$ and $K_{\phi \phi}^{(2) T}$. Using $\kappa=1$ instead leads, besides $\zeta_{2} \beta_{0}$ terms with $P_{\mathrm{qq}}^{(0)}(x)$ and $P_{\mathrm{qq}}^{(0)}(x) \ln x$, to the same spurious $\zeta_{2} C_{F}^{3}$ contribution as found in the analytic continuation in Ref. [12]. 
There is no obvious reason why an NNLO imperfection of the $A C$ relation should lead to an offset proportional to $\beta_{0}$ and the lowest-order kernel, and why exactly the same relation should hold for two different kernels. Hence it seems very likely that a non-vanishing r.h.s. of Eq. (9) is genuine and analogous to the 'Crewther discrepancy' between the Gross-Llewellyn Smith sum rule in DIS and the Adler function in $e^{+} e^{-}$annihilation observed and discussed in Ref. [23], see also the all-order proof and the recent explicit fourth-order calculation in Refs. [24].

Therefore, if one tried to fix the so far unknown $\zeta_{2}$ terms of the off-diagonal splitting functions by imposing Eq. (4) for $K_{1 \phi}^{(2)}, K_{\phi 1}^{(2)}$ and their timelike counterparts, one should find an offset proportional to $\beta_{0}$ in the known second moments which is then compensated by right-hand-sides analogous to that of Eq. (9). Carrying out these calculations indeed leads to

$$
\begin{aligned}
& {\left[A C_{0}\left[K_{1 \phi}^{(2)}\right]-K_{T \phi}^{(2)}\right]_{N=2}=\zeta_{2} \beta_{0} C_{F}\left(-\frac{212}{3} C_{A}-16 C_{F}\right),} \\
& {\left[A C_{0}\left[K_{\phi 1}^{(2)}\right]-K_{\phi T}^{(2)}\right]_{N=2}=\zeta_{2} \beta_{0} n_{f}\left(9 C_{A}-\frac{38}{3} C_{F}+4 \beta_{0}\right) .}
\end{aligned}
$$

For the required generalization of these relations to all values of $N$, we consider also the $\kappa=1$ continuation which, while again leading to spurious non- $\beta_{0}$ terms, appears to provide the right correction terms for Eq. (10),

$$
A C_{0}\left[K_{1 \phi}^{(2)}\right]-K_{T \phi}^{(2)}=-6 \zeta_{2} \beta_{0} P_{\mathrm{gq}}^{(0)}(x)\left[2 C_{A}(1-\ln x)+C_{F}\right],
$$

as well as the $\beta_{0}^{2}$ part of Eq. (11). We assume that also the first two terms on the r.h.s. of Eq. (11) correspond a to combination of $P_{\mathrm{qg}}^{(0)}$ and $P_{\mathrm{qg}}^{(0)} \ln x$. (Poly-) logarithms with higher weight cannot occur since this is an $n_{f}$ contribution and thus restricted to an overall weight of three. Imposing also other constraints discussed below we arrive at

$$
A C_{0}\left[K_{\phi 1}^{(2)}\right]-K_{\phi T}^{(2)}=\zeta_{2} \beta_{0} P_{\mathrm{qg}}^{(0)}(x)\left[8\left(C_{A}-C_{F}\right)-12 \ln x\left(C_{A}-2 C_{F}\right)+6 \beta_{0}\right] .
$$

Also the remaining uncertainty of the coefficients of $\ln x$ in this relation will be addressed below.

We are now ready to present the (only marginally provisional) results for the NNLO timelike splitting functions. For completeness we first recall the corresponding LO and NLO results:

$$
\begin{aligned}
& P_{\mathrm{qg}}^{(0) T}(x)=P_{\mathrm{qg}}^{(0) S}(x)=2 n_{f} p_{\mathrm{qg}}(x)=2 n_{f}\left(1-2 x+2 x^{2}\right), \\
& P_{\mathrm{gq}}^{(0) T}(x)=P_{\mathrm{gq}}^{(0) S}(x)=2 C_{F} p_{\mathrm{gq}}(x)=2 C_{F}\left(2 x^{-1}-2+x\right)
\end{aligned}
$$

and

$$
\begin{aligned}
& P_{\mathrm{qg}}^{(1) T}(x)=4 C_{F} n_{f}\left(-6+23 / 2 x-10 x^{2}-\left(5 / 2-2 x-2 x^{2}\right) \mathrm{H}_{0}-\left(1-2 x+4 x^{2}\right) \mathrm{H}_{0,0}+2 \mathrm{H}_{1}\right. \\
& \left.\quad+2 p_{\mathrm{qg}}(x)\left(-\zeta_{2}+\mathrm{H}_{1} / 2-3 \mathrm{H}_{1,0}-\mathrm{H}_{1,1}+\mathrm{H}_{2}\right)\right)-4 / 3 n_{f}^{2}\left(2+2 p_{\mathrm{qg}}(x)\left(2 / 3+\mathrm{H}_{0}-\mathrm{H}_{1}\right)\right) \\
& \quad+4 C_{A} n_{f}\left(-\left(20 x^{-1}-13+95 x-178 x^{2}\right) / 9-4 \zeta_{2} x-\left(4+34 x+4 x^{2}\right) / 3 \mathrm{H}_{0}\right. \\
& \left.\quad+(2+12 x) \mathrm{H}_{0,0}-2 \mathrm{H}_{1}-2 p_{\mathrm{qg}}(-x) \mathrm{H}_{-1,0}+2 p_{\mathrm{qg}}(x)\left(2 \mathrm{H}_{1,0}-5 / 6 \mathrm{H}_{1}+\mathrm{H}_{1,1}-2 \mathrm{H}_{2}\right)\right),(16) \\
& P_{\mathrm{gq}}^{(1) T}(x)=4 C_{F}^{2}\left((9 x-1) / 2-(8-x / 2) \mathrm{H}_{0}+(2-x) \mathrm{H}_{0,0}-2 x \mathrm{H}_{1}+2 p_{\mathrm{gq}}(x)\left(2 \mathrm{H}_{1,0}+\mathrm{H}_{1,1}\right.\right. \\
& \left.\left.\quad-2 \mathrm{H}_{2}\right)\right)+4 C_{F} C_{A}\left(+17 / 9 x^{-1}+5-x-44 / 9 x^{2}+4 \zeta_{2}-\left(6 x^{-1}-8-9 x-8 / 3 x^{2}\right) \mathrm{H}_{0}\right. \\
& \left.\quad-\left(8 x^{-1}+4+6 x\right) \mathrm{H}_{0,0}+2 x \mathrm{H}_{1}-2 p_{\mathrm{gq}}(-x) \mathrm{H}_{-1,0}-2 p_{\mathrm{gq}}(x)\left(3 \mathrm{H}_{1,0}+\mathrm{H}_{1,1}-\mathrm{H}_{2}\right)\right) .
\end{aligned}
$$


$H_{\vec{m}_{1}}$ are the harmonic polylogarithms (HPLs) as defined in Ref. [25] with $H_{0,1,0,1}(x) \equiv H_{2,2}$ etc. Our new third-order contributions to Eq. (2) are given by

$$
\begin{aligned}
& P_{\mathrm{qg}}^{(2) T}(x)= \\
& C_{F}^{2} n_{f}\left(-227 / 4-1967 / 2 x+1069 x^{2}-\left(288-352 x+160 x^{2}\right) \mathrm{H}_{1,0,0}+\left(34-32 x-4 x^{2}\right) \mathrm{H}_{1,1}\right. \\
& -\left(240-64 x+256 x^{2}\right) \mathrm{H}_{-2,0}-\left(180-192 x+144 x^{2}\right) \mathrm{H}_{1,1,0}-\left(176-288 x+416 x^{2}\right) \mathrm{H}_{2,0,0} \\
& -\left(124-64 x+48 x^{2}\right) \mathrm{H}_{1,2}-\left(120-240 x+288 x^{2}\right) \mathrm{H}_{2,1,0}-\left(104-208 x+224 x^{2}\right) \mathrm{H}_{2,2} \\
& -\left(96-64 x+256 x^{2}\right) \mathrm{H}_{-3,0}-\left(76-64 x+48 x^{2}\right) \mathrm{H}_{1,1,1}-\left(56-112 x+128 x^{2}\right) \mathrm{H}_{2,1,1} \\
& -\left(40-144 x+160 x^{2}\right) \mathrm{H}_{0,0} \zeta_{2}-\left(28+144 x-16 x^{2}\right) \mathrm{H}_{3}-\left(2-116 x+324 x^{2}\right) \mathrm{H}_{2} \\
& +\left(156-816 x-640 x^{2}\right) \zeta_{3}+\left(12+272 x-176 x^{2}\right) \mathrm{H}_{0} \zeta_{2}+\left(16-256 x+128 x^{2}\right) \mathrm{H}_{0,0,0,0} \\
& +\left(32+128 x+256 x^{2}\right) \mathrm{H}_{-2,0,0}+\left(36-584 x-192 x^{2}\right) \mathrm{H}_{0,0,0}+\left(64+320 x+256 x^{2}\right) \mathrm{H}_{-1,0,0} \\
& +\left(40-304 x+160 x^{2}\right) \mathrm{H}_{0} \zeta_{3}+\left(56-1904 / 5 x+352 / 5 x^{2}\right) \zeta_{2}^{2}+\left(64-128 x+192 x^{2}\right) \mathrm{H}_{3,1} \\
& +\left(64+64 x+128 x^{2}\right)\left(\mathrm{H}_{-2} \zeta_{2}+2 \mathrm{H}_{-2,-1,0}\right)-\left(496+240 x-224 x^{2}\right) \mathrm{H}_{-1,0} \\
& +\left(92-1143 x+110 x^{2}\right) \mathrm{H}_{0}+\left(96-192 x+352 x^{2}\right) \mathrm{H}_{3,0}+\left(104-144 x+224 x^{2}\right) \mathrm{H}_{2} \zeta_{2} \\
& +\left(104-80 x+16 x^{2}\right) \mathrm{H}_{2,1}+\left(106-564 x+564 x^{2}\right) \zeta_{2}+\left(107-42 x-76 x^{2}\right) \mathrm{H}_{0,0} \\
& +\left(108-64 x-80 x^{2}\right) \mathrm{H}_{1} \zeta_{2}+\left(128+160 x+32 x^{2}\right)\left(\mathrm{H}_{-1} \zeta_{2}+2 \mathrm{H}_{-1,-1,0}\right) \\
& +\left(180-480 x+318 x^{2}\right) \mathrm{H}_{1}+\left(184-128 x-112 x^{2}\right) \mathrm{H}_{2,0}+\left(294-560 x+468 x^{2}\right) \mathrm{H}_{1,0} \\
& +\left(8-16 x+32 x^{2}\right) \mathrm{H}_{4}-96 p_{\mathrm{qg}}(-x)\left(\mathrm{H}_{-1,-2,0}+2 \mathrm{H}_{-1,-1,0,0}+\mathrm{H}_{-1,0} \zeta_{2}-11 / 6 \mathrm{H}_{-1,0,0,0}\right) \\
& +16 p_{\mathrm{qg}}(x)\left(31 \mathrm{H}_{1} \zeta_{3}-6 \mathrm{H}_{1,-2,0}-7 \mathrm{H}_{1,0} \zeta_{2}+21 \mathrm{H}_{1,0,0,0}-\mathrm{H}_{1,1} \zeta_{2}+\mathrm{H}_{1,1,0,0}+3 \mathrm{H}_{1,1,1,0}\right. \\
& \left.\left.+2 \mathrm{H}_{1,1,1,1}+5 \mathrm{H}_{1,1,2}+19 \mathrm{H}_{1,2,0}+4 \mathrm{H}_{1,2,1}+5 \mathrm{H}_{1,3}\right)\right) \\
& +C_{A} C_{F} n_{f}\left(17597 / 36-220 / 3 x^{-1}+2659 / 6 x-3092 / 3 x^{2}-\left(288+448 x+64 x^{2}\right) \mathrm{H}_{-1,-1,0}\right. \\
& -\left(592 / 3-1352 / 3 x+192 x^{2}\right) \mathrm{H}_{2,1}-\left(2422 / 27-30203 / 27 x+17918 / 27 x^{2}\right) \mathrm{H}_{0} \\
& -\left(188-32 x^{-1}-272 x+120 x^{2}\right) \mathrm{H}_{1} \zeta_{2}-\left(1007 / 9+6254 / 9 x-2444 / 9 x^{2}\right) \mathrm{H}_{0,0} \\
& -\left(160+160 x+320 x^{2}\right) \mathrm{H}_{3,0}-\left(176-192 x+384 x^{2}\right) \mathrm{H}_{3,1}-\left(96+160 x-32 x^{2}\right) \mathrm{H}_{-1} \zeta_{2} \\
& -\left(64+32 x+128 x^{2}\right)\left(\mathrm{H}_{-2} \zeta_{2}+2 \mathrm{H}_{-2,-1,0}\right)+\left(28 / 3+2032 / 3 x+280 / 3 x^{2}\right) \mathrm{H}_{2,0} \\
& -\left(488 / 9-304 / 9 x^{-1}-868 / 9 x+1904 / 9 x^{2}\right) \mathrm{H}_{1,1}-\left(148 / 3-1832 / 3 x-640 / 3 x^{2}\right) \mathrm{H}_{0,0,0} \\
& -\left(48+64 x+64 x^{2}\right) \mathrm{H}_{-1,2}-(32+64 x) \mathrm{H}_{0,0,0,0}-\left(16+96 x^{-1}+504 x-368 x^{2}\right) \mathrm{H}_{1,0,0} \\
& -\left(44 / 5-2632 / 5 x+128 x^{2}\right) \zeta_{2}^{2}-\left(56-16 x+160 x^{2}\right) \mathrm{H}_{0} \zeta_{3}+\left(16-80 x-144 x^{2}\right) \mathrm{H}_{-1,0,0} \\
& +\left(32-256 x+256 x^{2}\right) \mathrm{H}_{0,0} \zeta_{2}+\left(64+96 x+128 x^{2}\right) \mathrm{H}_{4}+\left(28 / 9+4540 / 9 x-2620 / 3 x^{2}\right) \zeta_{2} \\
& +\left(268 / 3-32 x^{-1}-608 / 3 x+728 / 3 x^{2}\right) \mathrm{H}_{1,2}+\left(-260 / 3-176 / 3 x-8 x^{2}\right) \mathrm{H}_{0} \zeta_{2} \\
& +\left(2788 / 27-80 / 27 x^{-1}+19660 / 27 x-19970 / 27 x^{2}\right) \mathrm{H}_{1}+\left(388 / 3+256 / 3 x-40 / 3 x^{2}\right) \mathrm{H}_{3} \\
& +\left(136-1040 x+672 x^{2}\right) \mathrm{H}_{2,0,0}+\left(136-368 x+320 x^{2}\right) \mathrm{H}_{2,1,1}-\left(200-592 x+512 x^{2}\right) \mathrm{H}_{2} \zeta_{2} \\
& +\left(424 / 3-32 / 3 x^{-1}-536 / 3 x+544 / 3 x^{2}\right) \mathrm{H}_{1,1,1}+\left(2204 / 9-3772 / 9 x+3176 / 3 x^{2}\right) \mathrm{H}_{2} \\
& +\left(452 / 3-32 x^{-1}-1024 / 3 x+1048 / 3 x^{2}\right) \mathrm{H}_{1,1,0}+\left(168-624 x+448 x^{2}\right) \mathrm{H}_{2,2} \\
& +\left(184-656 x+512 x^{2}\right) \mathrm{H}_{2,1,0}+\left(144+32 x+384 x^{2}\right) \mathrm{H}_{-3,0}+\left(336+16 x+160 x^{2}\right) \mathrm{H}_{-2,0} \\
& +\left(1340 / 3+64 x^{-1}+4904 / 3 x+488 x^{2}\right) \zeta_{3}-\left(212-304 / 3 x^{-1}-2036 / 3 x+3284 / 3 x^{2}\right) \mathrm{H}_{1,0} \\
& +\left(672+544 x-16 x^{2}\right) \mathrm{H}_{-1,0}-\left(96 x+128 x^{2}\right) \mathrm{H}_{-2,0,0}-8 p_{\mathrm{qg}}(-x)\left(10 \mathrm{H}_{-1,-2,0}-8 \mathrm{H}_{-1,-1,0,0}\right.
\end{aligned}
$$


$\left.-10 \mathrm{H}_{-1,-1} \zeta_{2}+5 \mathrm{H}_{-1} \zeta_{3}-12 \mathrm{H}_{-1,-1,-1,0}+4 \mathrm{H}_{-1,-1,2}+3 \mathrm{H}_{-1,0,0,0}-12 \mathrm{H}_{-1,2,0}-4 \mathrm{H}_{-1,2,1}\right)$

$-8 p_{\mathrm{qg}}(x)\left(99 \mathrm{H}_{1} \zeta_{3}-10 \mathrm{H}_{1,-2,0}-10 \mathrm{H}_{1,0} \zeta_{2}+7 \mathrm{H}_{1,0,0,0}-4 \mathrm{H}_{1,1} \zeta_{2}-26 \mathrm{H}_{1,1,0,0}+2 \mathrm{H}_{1,1,1,0}\right.$

$\left.+8 \mathrm{H}_{1,1,1,1}+6 \mathrm{H}_{1,1,2}+30 \mathrm{H}_{1,2,0}+4 \mathrm{H}_{1,2,1}-14 \mathrm{H}_{1,3}\right)$ )

$+C_{A}^{2} n_{f}\left(2057 / 9+2092 / 81 x^{-1}-672 x+53753 / 81 x^{2}-\left(196+2416 / 3 x-328 / 3 x^{2}\right) \zeta_{3}\right.$

$-\left(3940 / 27-752 / 27 x^{-1}+8416 / 27 x-7448 / 27 x^{2}\right) \mathrm{H}_{1}+\left(288 x-96 x^{2}\right)\left(\mathrm{H}_{2,1,0}+\mathrm{H}_{2,2}\right)$

$-\left(1262 / 9-112 / 9 x^{-1}-4828 / 9 x+2900 / 3 x^{2}\right) \mathrm{H}_{2}-\left(100-64 / 3 x^{-1}+312 x-72 x^{2}\right) \mathrm{H}_{2,0}$

$-\left(352 / 3+64 / 3 x^{-1}+704 / 3 x+80 x^{2}\right) \mathrm{H}_{-1,2}-\left(100+776 x-96 x^{2}\right) \mathrm{H}_{4}-\left(64+128 x^{2}\right) \mathrm{H}_{-2,2}$

$-\left(2996 / 27-40 x^{-1}+4832 / 27 x-5044 / 9 x^{2}\right) \mathrm{H}_{0}-\left(104-432 x+32 x^{2}\right) \mathrm{H}_{-2,0,0}$

$-\left(80-256 x+192 x^{2}\right) \mathrm{H}_{2,1,1}-\left(598 / 9+128 / 9 x^{-1}+3508 / 9 x+7208 / 9 x^{2}\right) \mathrm{H}_{0,0}$

$-\left(196 / 3-32 / 3 x^{-1}-344 / 3 x+400 / 3 x^{2}\right) \mathrm{H}_{1,1,1}+\left(168-432 x+384 x^{2}\right) \mathrm{H}_{2} \zeta_{2}$

$-\left(310 / 3-544 / 9 x^{-1}-308 x+536 / 3 x^{2}\right) \zeta_{2}-(56-496 x) \mathrm{H}_{-3,0}+(64-1264 x) \mathrm{H}_{0,0,0,0}$

$-\left(152 / 3+32 / 3 x^{-1}+256 / 3 x+248 x^{2}\right) \mathrm{H}_{-1,0}-\left(28-632 x+96 x^{2}\right) \mathrm{H}_{0,0} \zeta_{2}$

$+\left(96+64 / 3 x^{-1}-484 / 3 x+824 / 3 x^{2}\right) \mathrm{H}_{0} \zeta_{2}+\left(134 / 9-128 / 9 x^{-1}-700 / 9 x+652 / 3 x^{2}\right) \mathrm{H}_{1,1}$

$+\left(16-352 x-64 x^{2}\right) \mathrm{H}_{-2,-1,0}+\left(728 / 3+224 / 3 x^{-1}+188 / 3 x-304 / 3 x^{2}\right) \mathrm{H}_{1,0,0}$

$+\left(64 / 3-128 / 3 x^{-1}+2680 / 3 x-192 x^{2}\right) \mathrm{H}_{0,0,0}+\left(64 / 3-64 / 3 x^{-1}+292 x+120 x^{2}\right) \mathrm{H}_{3}$

$+\left(34+252 / 5 x+704 / 5 x^{2}\right) \zeta_{2}^{2}+\left(160 / 3+32 x^{-1}+304 / 3 x-472 / 3 x^{2}\right)\left(\mathrm{H}_{1,1,0}+\mathrm{H}_{1,2}\right)$

$+\left(184 / 3+64 / 3 x^{-1}-344 / 3 x+832 / 3 x^{2}\right) \mathrm{H}_{-2,0}+\left(64-96 x+160 x^{2}\right) \mathrm{H}_{3,0}$

$+\left(440 / 3-64 / 3 x^{-1}-664 / 3 x+464 / 3 x^{2}\right) \mathrm{H}_{1} \zeta_{2}+\left(72-176 x+96 x^{2}\right) \mathrm{H}_{-2} \zeta_{2}$

$+\left(92+408 x-32 x^{2}\right) \mathrm{H}_{2,0,0}+\left(320 / 3-64 / 3 x^{-1}+112 / 3 x-208 x^{2}\right) \mathrm{H}_{-1,-1,0}$

$+\left(128+32 / 3 x^{-1}-400 x+640 / 3 x^{2}\right) \mathrm{H}_{2,1}+(16+960 x) \mathrm{H}_{0} \zeta_{3}+\left(176-192 x+320 x^{2}\right) \mathrm{H}_{3,1}$

$+\left(512 / 3+32 / 3 x^{-1}+760 / 3 x-24 x^{2}\right) \mathrm{H}_{-1} \zeta_{2}-\left(562 / 9+8 / 9 x^{-1}-92 / 9 x-704 / 3 x^{2}\right) \mathrm{H}_{1,0}$

$-\left(872 / 3-128 / 3 x^{-1}+520 / 3 x-304 / 3 x^{2}\right) \mathrm{H}_{-1,0,0}+8 p_{\mathrm{qg}}(-x)\left(17 \mathrm{H}_{-1} \zeta_{3}+14 \mathrm{H}_{-1,-2,0}\right.$

$-26 \mathrm{H}_{-1,-1} \zeta_{2}-12 \mathrm{H}_{-1,-1,-1,0}+8 \mathrm{H}_{-1,-1,0,0}+20 \mathrm{H}_{-1,-1,2}+14 \mathrm{H}_{-1,0} \zeta_{2}+\mathrm{H}_{-1,0,0,0}$

$\left.-8 \mathrm{H}_{-1,2,0}-4 \mathrm{H}_{-1,2,1}\right)+8 p_{\mathrm{qg}}(x)\left(31 \mathrm{H}_{1} \zeta_{3}-2 \mathrm{H}_{1,-2,0}+6 \mathrm{H}_{1,0} \zeta_{2}-15 \mathrm{H}_{1,0,0,0}-2 \mathrm{H}_{1,1} \zeta_{2}\right.$

$\left.\left.-20 \mathrm{H}_{1,1,0,0}-4 \mathrm{H}_{1,1,1,0}+4 \mathrm{H}_{1,1,1,1}-4 \mathrm{H}_{1,1,2}-4 \mathrm{H}_{1,2,1}-16 \mathrm{H}_{1,3}\right)\right)$

$+C_{F} n_{f}^{2}\left(-4847 / 54+200 / 27 x^{-1}-2375 / 27 x+4066 / 27 x^{2}-(32-64 x) \mathrm{H}_{0,0,0,0}\right.$

$-\left(416 / 9-376 / 9 x+88 / 3 x^{2}\right) \mathrm{H}_{2}+\left(1684 / 27+512 / 27 x^{-1}-4022 / 27 x-4480 / 27 x^{2}\right) \mathrm{H}_{0}$

$-\left(808 / 27-560 / 27 x+248 / 27 x^{2}\right) \mathrm{H}_{1}+\left(902 / 9+128 / 9 x^{-1}+956 / 9 x+1400 / 9 x^{2}\right) \mathrm{H}_{0,0}$

$-\left(64 / 3-128 / 3 x+176 / 3 x^{2}\right) \mathrm{H}_{3}+\left(272 / 9-64 / 9 x^{-1}+8 / 9 x+32 x^{2}\right) \zeta_{2}$

$+\left(48-64 / 9 x^{-1}-496 / 9 x^{2}\right) \mathrm{H}_{-1,0}+\left(344 / 9-376 / 9 x+328 / 9 x^{2}\right) \mathrm{H}_{1,1}$

$+\left(80-160 x+176 x^{2}\right) / 3 \mathrm{H}_{2,0}-\left(32-112 x-96 x^{2}\right) / 3 \mathrm{H}_{0,0,0}+\left(64-128 x+144 x^{2}\right) / 3 \mathrm{H}_{2,1}$

$+\left(32-64 x+64 / 3 x^{2}\right) \mathrm{H}_{-2,0}+\left(80-352 x+144 x^{2}\right) / 3 \mathrm{H}_{0} \zeta_{2}+\left(304 / 3-608 / 3 x+160 x^{2}\right) \zeta_{3}$

$\left.+\left(192-248 x+232 x^{2}\right) / 3 \mathrm{H}_{1,0}-8 / 3 p_{\mathrm{qg}}(x)\left(3 \mathrm{H}_{1} \zeta_{2}+5 \mathrm{H}_{1,2}+7 \mathrm{H}_{1,1,0}+5 \mathrm{H}_{1,1,1}-12 \mathrm{H}_{1,0,0}\right)\right)$

$+C_{A} n_{f}^{2}\left(-14 / 9-44 / 9 x^{-1}-1216 / 9 x+916 / 9 x^{2}-\left(40-352 / 3 x+272 / 3 x^{2}\right) \zeta_{3}\right.$

$-\left(296 / 9+64 / 9 x^{-1}+416 / 9 x+536 / 9 x^{2}\right) \mathrm{H}_{0,0}-\left(32-96 x+224 / 3 x^{2}\right) \mathrm{H}_{2,1}$

$-(64 / 3+640 / 3 x) \mathrm{H}_{0,0,0}+\left(44 / 27-256 / 27 x^{-1}+752 / 27 x-2212 / 27 x^{2}\right) \mathrm{H}_{0}$ 


$$
\begin{aligned}
& +\left(100 / 3+32 / 9 x^{-1}+208 / 3 x-16 / 9 x^{2}\right) \zeta_{2}-\left(24-112 / 3 x+112 / 3 x^{2}\right) \mathrm{H}_{0} \zeta_{2} \\
& -\left(320 / 9-32 / 9 x^{-1}-544 / 9 x+200 / 3 x^{2}\right) \mathrm{H}_{1,1}-\left(64 / 3-128 / 3 x+64 / 3 x^{2}\right) \mathrm{H}_{-2,0} \\
& +\left(16 / 9+32 / 3 x^{-1}+496 / 9 x-664 / 9 x^{2}\right) \mathrm{H}_{1,0}+\left(356 / 9-1360 / 9 x+632 / 9 x^{2}\right) \mathrm{H}_{2} \\
& +\left(592-320 x^{-1}-1232 x+2632 x^{2}\right) / 27 \mathrm{H}_{1}+\left(104 / 3-16 x+176 / 3 x^{2}\right) \mathrm{H}_{3} \\
& -\left(144-32 x^{-1}-96 x-512 x^{2}\right) / 9 \mathrm{H}_{-1,0}+\left(8+80 x-16 x^{2}\right) \mathrm{H}_{2,0}+32 / 3 p_{\mathrm{qg}}(-x)\left(2 \mathrm{H}_{-1} \zeta_{2}\right. \\
& \left.\left.+2 \mathrm{H}_{-1,-1,0}+\mathrm{H}_{-1,0,0}-\mathrm{H}_{-1,2}\right)-8 / 3 p_{\mathrm{qg}}(x)\left(4 \mathrm{H}_{1} \zeta_{2}+19 \mathrm{H}_{1,0,0}+2 \mathrm{H}_{1,1,0}-5 \mathrm{H}_{1,1,1}+2 \mathrm{H}_{1,2}\right)\right) \\
& +n_{f}^{3}\left(16 / 9+8 / 3\left[\mathrm{H}_{0}-\mathrm{H}_{1}+p_{\mathrm{qg}}(x)\left(\zeta_{2}-1+2 / 3\left(\mathrm{H}_{0}-\mathrm{H}_{1}\right)+\mathrm{H}_{0,0}-\mathrm{H}_{1,0}+\mathrm{H}_{1,1}-\mathrm{H}_{2}\right)\right]\right)
\end{aligned}
$$

and

$$
\begin{aligned}
& P_{\mathrm{gq}}^{(2) T}(x)= \\
& C_{F}^{3}\left(-1915 / 2+794 x^{-1}+731 / 4 x-\left(464-320 x^{-1}-200 x\right) \mathrm{H}_{2} \zeta_{2}-(290+251 x) \mathrm{H}_{0,0}\right. \\
& -\left(352-144 x^{-1}-252 x\right) \mathrm{H}_{1} \zeta_{2}-\left(264-180 x^{-1}-140 x\right) \mathrm{H}_{1,0}-\left(240-256 x^{-1}-120 x\right) \mathrm{H}_{3,1} \\
& -\left(230-228 x^{-1}-16 x\right) \mathrm{H}_{1}-\left(224+256 x^{-1}-148 x\right) \zeta_{2}-\left(176-128 x^{-1}-88 x\right) \mathrm{H}_{3,0} \\
& -\left(776 / 5-1216 / 5 x^{-1}-84 / 5 x\right) \zeta_{2}^{2}-(128-64 x) \mathrm{H}_{4}-(104-40 x) \mathrm{H}_{2,0}+(248-28 x) \mathrm{H}_{3} \\
& -\left(96+720 x^{-1}+196 x\right) \zeta_{3}-\left(80+256 x^{-1}-208 x\right) \mathrm{H}_{-1,0}-\left(80-128 x^{-1}+200 x\right) \mathrm{H}_{0} \zeta_{3} \\
& -\left(72-60 x^{-1}-80 x\right) \mathrm{H}_{1,1}-(72-56 x) \mathrm{H}_{2,1}-(64+32 x)\left(\mathrm{H}_{-1} \zeta_{2}+2 \mathrm{H}_{-1,-1,0}\right) \\
& -(64+96 x) \mathrm{H}_{-3,0}-(64+64 x) \mathrm{H}_{0,0} \zeta_{2}-\left(64+128 x^{-1}+64 x\right) \mathrm{H}_{-2,0,0}+(208-140 x) \mathrm{H}_{2} \\
& -\left(32-48 x^{-1}-4 x\right) \mathrm{H}_{1,1,0}-(32-96 x) \mathrm{H}_{0,0,0,0}-\left(24+192 x^{-1}+20 x\right) \mathrm{H}_{0} \zeta_{2} \\
& +\left(197+208 x^{-1}+29 x\right) \mathrm{H}_{0}+(16-52 x) \mathrm{H}_{1,1,1}+\left(128+128 x^{-1}+96 x\right)\left(\mathrm{H}_{-2} \zeta_{2}+2 \mathrm{H}_{-2,-1,0}\right) \\
& +\left(128-192 x^{-1}-112 x\right) \mathrm{H}_{-2,0}+\left(128-48 x^{-1}-60 x\right) \mathrm{H}_{1,2}+\left(128+48 x^{-1}-36 x\right) \mathrm{H}_{1,0,0} \\
& +\left(80-64 x^{-1}-40 x\right) \mathrm{H}_{2,1,0}+\left(144-64 x^{-1}-72 x\right) \mathrm{H}_{2,2}+(160+40 x) \mathrm{H}_{0,0,0} \\
& -\left(16-128 x^{-1}-40 x\right) \mathrm{H}_{2,0,0}+\left(208-192 x^{-1}-104 x\right) \mathrm{H}_{2,1,1}+\left(224+96 x^{-1}+96 x\right) \mathrm{H}_{-1,0,0} \\
& +16 p_{\mathrm{gq}}(-x)\left(2 \mathrm{H}_{-1,-2,0}+4 \mathrm{H}_{-1,-1,0,0}-\mathrm{H}_{-1,0,0,0}\right)+16 p_{\mathrm{gq}}(x)\left(2 \mathrm{H}_{1} \zeta_{3}-2 \mathrm{H}_{1,-2,0}+4 \mathrm{H}_{1,0} \zeta_{2}\right. \\
& \left.\left.-6 \mathrm{H}_{1,0,0,0}+3 \mathrm{H}_{1,1} \zeta_{2}-6 \mathrm{H}_{1,1,0,0}+\mathrm{H}_{1,1,1,0}+2 \mathrm{H}_{1,1,1,1}-3 \mathrm{H}_{1,1,2}+\mathrm{H}_{1,2,0}-4 \mathrm{H}_{1,2,1}-8 \mathrm{H}_{1,3}\right)\right) \\
& +C_{A} C_{F}^{2}\left(1735 / 6+200 x^{-1}-4811 / 12 x-140 / 3 x^{2}-\left(336-448 x^{-1}-312 x\right) \mathrm{H}_{2,2}\right. \\
& -\left(2338 / 3-80 x^{-1}+441 x+1408 / 9 x^{2}\right) \mathrm{H}_{0,0}-\left(1760 / 3-336 x^{-1}-832 / 3 x+64 / 3 x^{2}\right) \mathrm{H}_{1,0,0} \\
& -\left(336-384 x^{-1}-216 x\right) \mathrm{H}_{2,1,0}-\left(8980 / 9+5800 / 9 x^{-1}+4576 / 9 x+352 / 3 x^{2}\right) \mathrm{H}_{2} \\
& -\left(976 / 3-904 / 3 x^{-1}-404 / 3 x+32 x^{2}\right) \mathrm{H}_{1,2}-\left(320-256 x^{-1}-208 x\right) \mathrm{H}_{2,0,0} \\
& -\left(272+832 x^{-1}+376 x\right) \mathrm{H}_{0} \zeta_{3}-\left(272-320 x^{-1}-184 x\right) \mathrm{H}_{2,1,1}-\left(208+448 x^{-1}+344 x\right) \mathrm{H}_{3,0} \\
& -\left(192+48 x^{-1}+72 x\right) \mathrm{H}_{-1,0,0}-\left(144-96 x^{-1}-128 x\right) \mathrm{H}_{-2,0}-\left(128-32 x^{-1}+16 x\right) \mathrm{H}_{-2} \zeta_{2} \\
& -\left(128+64 x^{-1}+96 x\right) \mathrm{H}_{-2,-1,0}-\left(256 / 3-88 x^{-1}-332 / 3 x+32 / 3 x^{2}\right) \mathrm{H}_{1,1,0} \\
& -\left(608 / 5+3664 / 5 x^{-1}+2144 / 5 x\right) \zeta_{2}^{2}-\left(88-192 x^{-1}+268 x\right) \mathrm{H}_{-1,0}-(32-48 x) \mathrm{H}_{-3,0} \\
& -\left(152 / 3-160 / 3 x^{-1}-232 / 3 x+32 / 3 x^{2}\right) \mathrm{H}_{1,1,1}+\left(552+264 x^{-1}+424 x+128 / 3 x^{2}\right) \zeta_{3} \\
& +\left(136 / 3+640 / 3 x^{-1}-680 / 3 x-32 x^{2}\right) \mathrm{H}_{3}-\left(160 / 9-848 / 9 x^{-1}+280 / 9 x+352 / 9 x^{2}\right) \mathrm{H}_{1,1} \\
& -\left(64+24 x^{-1}+32 x\right) \mathrm{H}_{-1} \zeta_{2}-\left(16+128 x^{-1}+40 x\right) \mathrm{H}_{0,0} \zeta_{2}-\left(16+320 x^{-1}+216 x\right) \mathrm{H}_{3,1} \\
& +\left(72+192 x^{-1}+128 x-32 / 3 x^{2}\right) \mathrm{H}_{0} \zeta_{2}+\left(368 / 3-268 / 3 x+64 / 3 x^{2}\right) \mathrm{H}_{0,0,0} \\
& +\left(424 / 3-464 / 3 x^{-1}+28 / 3 x+32 x^{2}\right) \mathrm{H}_{2,1}+\left(600-1280 / 3 x^{-1}+96 x+224 / 3 x^{2}\right) \mathrm{H}_{2,0}
\end{aligned}
$$




$$
\begin{aligned}
& +\left(488 / 3+620 / 9 x^{-1}-200 x-704 / 9 x^{2}\right) \mathrm{H}_{1,0}+\left(192+48 x^{-1}+128 x\right) \mathrm{H}_{-1,-1,0} \\
& +\left(272-608 x^{-1}-456 x\right) \mathrm{H}_{2} \zeta_{2}+\left(336+512 x^{-1}+376 x\right) \mathrm{H}_{4}+\left(160+48 x^{-1}+96 x\right) \mathrm{H}_{-1,2} \\
& -(32+32 x)\left(\mathrm{H}_{-2,0,0}-2 \mathrm{H}_{0,0,0,0}\right)+\left(40301 / 27+244 x^{-1}+18911 / 27 x+8932 / 27 x^{2}\right) \mathrm{H}_{0} \\
& +\left(672-1760 / 3 x^{-1}-180 x+224 / 3 x^{2}\right) \mathrm{H}_{1} \zeta_{2}+\left(3452 / 3+528 x^{-1}+436 / 3 x+1760 / 9 x^{2}\right) \zeta_{2} \\
& +\left(26008 / 27-39092 / 27 x^{-1}+3988 / 27 x+5908 / 27 x^{2}\right) \mathrm{H}_{1}+8 p_{\mathrm{gq}}(-x)\left(4 \mathrm{H}_{-1,3}+11 \mathrm{H}_{-1} \zeta_{3}\right. \\
& -18 \mathrm{H}_{-1,-1} \zeta_{2}-8 \mathrm{H}_{-1,2,0}-4 \mathrm{H}_{-1,2,1}-4 \mathrm{H}_{-1,-1,0,0}+12 \mathrm{H}_{-1,-1,2}+12 \mathrm{H}_{-1,0} \zeta_{2}+\mathrm{H}_{-1,0,0,0} \\
& \left.+10 \mathrm{H}_{-1,-2,0}-12 \mathrm{H}_{-1,-1,-1,0}\right)-8 p_{\mathrm{gq}}(x)\left(4 \mathrm{H}_{-2,2}+51 \mathrm{H}_{1} \zeta_{3}-10 \mathrm{H}_{1,1,0,0}-14 \mathrm{H}_{1,3}-4 \mathrm{H}_{1,2,1}\right. \\
& \left.\left.+3 \mathrm{H}_{1,0,0,0}+12 \mathrm{H}_{1,1} \zeta_{2}-6 \mathrm{H}_{1,-2,0}-2 \mathrm{H}_{1,0} \zeta_{2}+8 \mathrm{H}_{1,1,1,1}+2 \mathrm{H}_{1,1,2}+34 \mathrm{H}_{1,2,0}+14 \mathrm{H}_{1,1,1,0}\right)\right) \\
& +C_{A}^{2} C_{F}\left(-\left(12+752 / 3 x^{-1}-532 / 3 x+128 / 3 x^{2}\right) \mathrm{H}_{0} \zeta_{2}-\left(152+128 x^{-1}+92 x\right) \mathrm{H}_{4}\right. \\
& -\left(22270 / 27-31504 / 27 x^{-1}+688 / 27 x+1924 / 9 x^{2}\right) \mathrm{H}_{1}-\left(144+64 x^{-1}+8 x\right) \mathrm{H}_{-2} \zeta_{2} \\
& -\left(17798 / 27-3514 / 9 x^{-1}+3436 / 27 x-4136 / 9 x^{2}\right)-\left(400-160 x^{-1}-148 x+640 / 3 x^{2}\right) \zeta_{3} \\
& -\left(576-1504 / 3 x^{-1}+424 x+512 / 3 x^{2}\right) \mathrm{H}_{2,0}-\left(1292 / 3+32 / 3 x^{-1}+284 / 3 x+128 / 3 x^{2}\right) \mathrm{H}_{3} \\
& -\left(848 / 3-1856 / 3 x^{-1}+3344 / 3 x+224 / 3 x^{2}\right) \mathrm{H}_{0,0,0}+\left(572 / 5+1984 / 5 x^{-1}+314 x\right) \zeta_{2}^{2} \\
& -\left(32612 / 27-13384 / 27 x^{-1}+23630 / 27 x+12964 / 27 x^{2}\right) \mathrm{H}_{0}-(160-48 x) \mathrm{H}_{-2,-1,0} \\
& -\left(168+176 / 3 x^{-1}+160 x+32 / 3 x^{2}\right) \mathrm{H}_{-1} \zeta_{2}-\left(280-344 x^{-1}+32 x+64 x^{2}\right) \mathrm{H}_{1} \zeta_{2} \\
& -\left(848+5176 / 9 x^{-1}+2800 / 9 x+352 / 9 x^{2}\right) \zeta_{2}+\left(152-128 x^{-1}-156 x\right) \mathrm{H}_{0,0} \zeta_{2} \\
& -\left(1280 / 9+248 / 3 x^{-1}-1052 / 9 x-704 / 9 x^{2}\right) \mathrm{H}_{-1,0}-\left(128-512 x^{-1}-632 x\right) \mathrm{H}_{0,0,0,0} \\
& -\left(328 / 3+272 x^{-1}-544 / 3 x+128 / 3 x^{2}\right) \mathrm{H}_{-1,0,0}+\left(128-192 x^{-1}-112 x\right) \mathrm{H}_{2,1,0} \\
& +\left(384+576 x^{-1}+480 x\right)\left(\mathrm{H}_{3,0}+1 / 3 \mathrm{H}_{3,1}\right)-\left(296 / 3-448 / 3 x^{-1}+260 / 3 x+128 / 3 x^{2}\right) \mathrm{H}_{2,1} \\
& -\left(280 / 3+752 / 3 x^{-1}-928 / 3 x+256 / 3 x^{2}\right) \mathrm{H}_{-2,0}+\left(64-128 x^{-1}-80 x\right) \mathrm{H}_{2,1,1} \\
& -\left(16-352 / 3 x^{-1}+160 x-64 / 3 x^{2}\right) \mathrm{H}_{-1,-1,0}+\left(1300 / 3-1024 / 3 x^{-1}-1004 / 3 x\right) \mathrm{H}_{1,0,0} \\
& +\left(104 / 3-160 / 3 x^{-1}-76 / 3 x+32 / 3 x^{2}\right) \mathrm{H}_{1,1,1}+\left(48+320 x^{-1}+248 x\right) \mathrm{H}_{2} \zeta_{2} \\
& +\left(11848 / 9+3920 / 9 x^{-1}+7280 / 9 x+3688 / 9 x^{2}\right) \mathrm{H}_{0,0}+\left(112-256 x^{-1}-248 x\right) \mathrm{H}_{-3,0} \\
& +\left(368 / 3-424 / 3 x^{-1}-352 / 3 x+32 / 3 x^{2}\right) \mathrm{H}_{1,1,0}+\left(160+352 / 3 x^{-1}+80 x+64 / 3 x^{2}\right) \mathrm{H}_{-1,2} \\
& +\left(240-96 x^{-1}-136 x\right) \mathrm{H}_{-2,0,0}+\left(1160 / 9-4136 / 9 x^{-1}-604 / 9 x+760 / 3 x^{2}\right) \mathrm{H}_{1,0} \\
& +\left(64-256 x^{-1}-176 x\right) \mathrm{H}_{2,2}+\left(352+416 x^{-1}+240 x\right) \mathrm{H}_{0} \zeta_{3}+\left(232-160 x^{-1}-76 x\right) \mathrm{H}_{2,0,0} \\
& +\left(192-248 x^{-1}-72 x+32 x^{2}\right) \mathrm{H}_{1,2}+\left(6896 / 9+4664 / 9 x^{-1}+4220 / 9 x+1552 / 9 x^{2}\right) \mathrm{H}_{2} \\
& +\left(808 / 9-532 / 3 x^{-1}-440 / 9 x+560 / 9 x^{2}\right) \mathrm{H}_{1,1}-8 p_{\mathrm{gq}}(-x)\left(4 \mathrm{H}_{-2,2}-\mathrm{H}_{-1} \zeta_{3}+22 \mathrm{H}_{-1,-2,0}\right. \\
& -2 \mathrm{H}_{-1,-1} \zeta_{2}-12 \mathrm{H}_{-1,-1,-1,0}+12 \mathrm{H}_{-1,-1,0,0}-4 \mathrm{H}_{-1,-1,2}+10 \mathrm{H}_{-1,0} \zeta_{2}-21 \mathrm{H}_{-1,0,0,0} \\
& \left.-12 \mathrm{H}_{-1,2,0}-4 \mathrm{H}_{-1,2,1}+4 \mathrm{H}_{-1,3}\right)+8 p_{\mathrm{gq}}(x)\left(41 \mathrm{H}_{1} \zeta_{3}-6 \mathrm{H}_{1,-2,0}-8 \mathrm{H}_{1,0} \zeta_{2}+35 \mathrm{H}_{1,0,0,0}\right. \\
& \left.+6 \mathrm{H}_{1,1} \zeta_{2}+10 \mathrm{H}_{1,1,0,0}+12 \mathrm{H}_{1,1,1,0}+4 \mathrm{H}_{1,1,1,1}+8 \mathrm{H}_{1,1,2}+40 \mathrm{H}_{1,2,0}+4 \mathrm{H}_{1,2,1}+10 \mathrm{H}_{1,3}\right) \text { ) } \\
& +C_{F}^{2} n_{f}\left(-12803 / 27+14008 / 81 x^{-1}+30475 / 54 x-21784 / 81 x^{2}+(64-32 x) \mathrm{H}_{0,0,0,0}\right. \\
& -\left(204+128 / 3 x^{-1}-142 / 3 x+256 / 9 x^{2}\right) \mathrm{H}_{0,0}-\left(176 / 3+128 / 3 x^{-1}-160 / 3 x\right) \mathrm{H}_{0,0,0} \\
& -\left(484 / 27-448 / 27 x^{-1}-464 / 27 x\right) \mathrm{H}_{1}+\left(160 / 9-160 / 9 x^{-1}-104 / 9 x\right)\left(\mathrm{H}_{1,1}-2 \mathrm{H}_{2}\right) \\
& -\left(2330 / 27-200 / 9 x^{-1}-2494 / 27 x-3040 / 27 x^{2}\right) \mathrm{H}_{0}-8 / 3 p_{\mathrm{gq}}(x)\left(3 \mathrm{H}_{1} \zeta_{2}+5 \mathrm{H}_{1,0}\right. \\
& \left.+5 \mathrm{H}_{1,0,0}+\mathrm{H}_{1,1,0}-\mathrm{H}_{1,1,1}+\mathrm{H}_{1,2}+2 \mathrm{H}_{2,1}-4 \mathrm{H}_{3}\right) \text { ) }
\end{aligned}
$$




$$
\begin{aligned}
+ & C_{A} C_{F} n_{f}\left(332 / 27+3778 / 81 x^{-1}-2624 / 27 x+1892 / 81 x^{2}+\left(64-80 x^{-1}-40 x\right) \zeta_{3}\right. \\
& -\left(256 / 9-656 / 9 x^{-1}+464 / 9 x+128 / 9 x^{2}\right) \mathrm{H}_{0,0}-\left(160 / 9+160 / 9 x^{-1}+104 / 9 x\right) \mathrm{H}_{-1,0} \\
& -\left(160 / 9-160 / 9 x^{-1}-104 / 9 x\right) \mathrm{H}_{1,1}+\left(32 / 3+128 / 3 x^{-1}+128 / 3 x\right) \mathrm{H}_{0,0,0} \\
& +\left(136 / 9-160 / 9 x^{-1}-104 / 9 x\right) \mathrm{H}_{2}+\left(448 / 27-448 / 27 x^{-1}-392 / 27 x\right) \mathrm{H}_{1} \\
& +\left(1280 / 27+1156 / 27 x^{-1}+308 / 27 x+64 / 9 x^{2}\right) \mathrm{H}_{0}-\left(320 / 9-320 / 9 x^{-1}-136 / 9 x\right) \mathrm{H}_{1,0} \\
& -\left(32 / 3 x^{-1}+16 / 3 x\right) \mathrm{H}_{0} \zeta_{2}+\left(8 / 3-160 / 9 x^{-1}-176 / 9 x\right) \zeta_{2}+16 / 3 p_{\mathrm{gq}}(-x)\left(\mathrm{H}_{-2,0}\right. \\
& \left.\left.+\mathrm{H}_{-1,0,0}\right)+8 / 3 p_{\mathrm{gq}}(x)\left(6 \mathrm{H}_{1} \zeta_{2}+10 \mathrm{H}_{1,0,0}+2 \mathrm{H}_{1,1,0}-\mathrm{H}_{1,1,1}+\mathrm{H}_{2,1}-2 \mathrm{H}_{3}\right)\right) .
\end{aligned}
$$

Unlike their spacelike counterparts, the off-diagonal timelike splitting functions show a doublelogarithmic enhancement of higher-order terms not only for $x \rightarrow 1$, but also for $x \rightarrow 0$ [26] with

and

$$
\begin{aligned}
& P_{\mathrm{qg}}^{(1) T}(x)=4 C_{A F} n_{f} L_{1}^{2}+\left[\frac{44}{3} C_{A F}+\frac{8}{3}\left(C_{F}-n_{f}\right)\right] n_{f} L_{1}+O(1), \\
& P_{\mathrm{gq}}^{(1) T}(x)=-4 C_{A F} C_{F} L_{1}^{2}-8 C_{A F} C_{F} L_{1}+O(1)
\end{aligned}
$$

$$
\begin{aligned}
& x P_{\mathrm{qg}}^{(1) T}(x)=-\frac{80}{9} C_{A} n_{f}+O\left(x L_{0}^{2}\right), \\
& x P_{\mathrm{gq}}^{(1) T}(x)=-16 C_{F} C_{A} L_{0}^{2}-24 C_{F} C_{A} L_{0}+\frac{68}{9} C_{F} C_{A}+O\left(x L_{0}^{2}\right) .
\end{aligned}
$$

Here and below we use the abbreviations $L_{1}=-\mathrm{H}_{1}(x)=\ln (1-x), L_{0}=\mathrm{H}_{0}(x)=\ln x$ and $C_{A F}=$ $C_{A}-C_{F}$. The large- $x$ and small- $x$ limits of the new result (18) and (19) are given by

$$
\begin{aligned}
P_{\mathrm{qg}}^{(2) T}(x)= & \frac{4}{3} C_{A F}^{2} n_{f} L_{1}^{4}+\left[\frac{110}{9} C_{A F}^{2}+\frac{20}{9} C_{A F}\left(C_{F}-n_{f}\right)\right] n_{f} L_{1}^{3} \\
+ & {\left[\left(\frac{631}{9}-8 \zeta_{2}\right) C_{A F}^{2}+\left(\frac{652}{9}-16 \zeta_{2}\right) C_{A F} C_{F}-\frac{172}{9} C_{A F} n_{f}+\frac{4}{3}\left(C_{F}-n_{f}\right)^{2}\right] n_{f} L_{1}^{2} } \\
+ & {\left[\left(\frac{4156}{27}-\frac{176}{3} \zeta_{2}+16 \zeta_{3}\right) C_{A F}^{2}+\left(\frac{5914}{27}-84 \zeta_{2}+96 \zeta_{3}\right) C_{A F} C_{F}+\frac{424}{9} C_{F}^{2}\right.} \\
& \left.-\left(\frac{1672}{27}-\frac{32}{3} \zeta_{2}\right) C_{A F} n_{f}-\frac{392}{9} C_{F} n_{f}+\frac{40}{9} n_{f}^{2}-\frac{40}{3} \zeta_{2} C_{F}\left(C_{F}-n_{f}\right)\right] n_{f} L_{1}+O(1) \\
P_{\mathrm{gq}}^{(2) T}(x)= & \frac{4}{3} C_{F} C_{A F}^{2} L_{1}^{4}+\left[\frac{50}{9} C_{A F}^{2}-\frac{4}{9} C_{A F}\left(C_{F}-n_{f}\right)\right] C_{F} L_{1}^{3} \\
+ & {\left[\frac{52}{9} C_{A F} n_{f}-\left(\frac{334}{9}-8 \zeta_{2}\right) C_{A F}^{2}-\left(\frac{640}{9}-16 \zeta_{2}\right) C_{A F} C_{F}\right] C_{F} L_{1}^{2} } \\
+ & {\left[\left(-\frac{2774}{27}+\frac{8}{3} \zeta_{2}+80 \zeta_{3}\right) C_{A F}^{2}-\left(\frac{2360}{27}-\frac{148}{3} \zeta_{2}+48 \zeta_{3}\right) C_{A F} C_{F}\right.} \\
& \left.+\left(\frac{392}{27}-\frac{32}{3} \zeta_{2}\right) C_{A F} n_{f}+\frac{4}{3}\left(1+2 \zeta_{2}\right) C_{F}\left(C_{F}-n_{f}\right)\right] C_{F} L_{1}+O(1) \\
x P_{\mathrm{qg}}^{(2) T}(x)= & -\frac{64}{9} C_{A}^{2} n_{f} L_{0}^{3}-\left[\frac{64}{9} C_{A}^{2} n_{f}+\frac{32}{9}\left(C_{A}-2 C_{F}\right) n_{f}^{2}\right] L_{0}^{2} \\
+ & {\left[\left(40+\frac{64}{3} \zeta_{2}\right) C_{A}^{2} n_{f}-\frac{256}{27}\left(C_{A}-2 C_{F}\right) n_{f}^{2}\right] L_{0}+O(1) } \\
= & \frac{64}{3} C_{F} C_{A}^{2} L_{0}^{4}+\left[\frac{928}{9} C_{F} C_{A}^{2}+\frac{64}{9} C_{F} n_{f}\left(C_{A}-C_{F}\right)\right] L_{0}^{3} \\
+ & {\left[\left(40-64 \zeta_{2}\right) C_{F}^{2} C_{A}+\left(\frac{1960}{9}-64 \zeta_{2}\right) C_{F} C_{A}^{2}-\frac{64}{3} C_{F}^{2} n_{f}+\frac{328}{9} C_{F} C_{A} n_{f}\right] L_{0}^{2} } \\
x P_{\mathrm{gq}}^{(2) T}(x) & {\left[\left(\frac{13384}{27}-\frac{752}{3} \zeta_{2}+416 \zeta_{3}\right) C_{F} C_{A}^{2}+\left(244+192 \zeta_{2}-832 \zeta_{3}\right) C_{F}^{2} C_{A}\right.} \\
& \left.+\left(208-192 \zeta_{2}+128 \zeta_{3}\right) C_{F}^{3}+\left(\frac{1156}{27}-\frac{32}{3} \zeta_{2}\right) C_{F} C_{A} n_{f}+\frac{200}{9} C_{F}^{2} n_{f}\right] L_{0}+O(1)
\end{aligned}
$$


The leading logarithms in all four large- $x$ limits above are identical to those of the corresponding spacelike splitting functions, in agreement with the all-order prediction in Ref. [27]. As in the spacelike case [11], all double-logarithmic contributions to these equations vanish for $C_{A}=C_{F}$. Only one coefficient, that of $\ln ^{1}(1-x)$ in Eq. (24), does not vanish in the supersymmetric limit $C_{A}=C_{F}=n_{f}$. Also this feature is analogous to the spacelike case discussed in Ref. [11]. Eq. (25) is in complete agreement with the corresponding result of Ref. [28], see also Ref. [29]. This would not be the case if a term with $\ln (1-x)$ were present on the right-hand-side of Eq. (12).

The coefficients of the leading small- $x$ logarithms of $P_{\mathrm{qg}}^{T}$ and $P_{\mathrm{gq}}^{T}$ at NLO and NNLO are larger and smaller, respectively, by a factor $C_{A} / C_{F}$ than those of $P_{\mathrm{qq}}^{T}$ and $P_{\mathrm{gg}}^{T}$ in Eqs. (13) - (15) of Ref. [13]. For $P_{\mathrm{gq}}^{T}$ and $P_{\mathrm{gg}}^{T}$ this relation and the corresponding coefficients have been derived to all orders in Refs. [26]. Unlike at NLO, $P_{\mathrm{qq}}^{T}$ and $P_{\mathrm{qg}}^{T}$ are suppressed by only one power of $\ln (1-x)$ relative to $P_{\mathrm{gq}}^{T}$ and $P_{\mathrm{gg}}^{T}$ at NNLO, and presumably some or all higher orders.

We now turn to the additional constraints mentioned above Eq. (13). These are provided by the well-known supersymmetric relations for the choice $C_{A}=C_{F}=n_{f}$ of the colour factors leading to a $\mathcal{N}=1$ supersymmetric theory. On the one hand, we can investigate the combinations

$$
\Delta_{\mathrm{A}}^{(n)}(x)=P_{\mathrm{qq}}^{(n) A}(x)+P_{\mathrm{gq}}^{(n) A}(x)-P_{\mathrm{qg}}^{(n) A}(x)-P_{\mathrm{gg}}^{(n) A}(x) \quad \text { with } \quad A=S, T
$$

which vanish at LO, while at NLO and $x<1$ these quantities are given by

$$
\begin{aligned}
& \Delta_{\mathrm{S}}^{(1)}(x)=\frac{8}{3} x^{-1}+\left(4-8 x-16 x^{2}\right) \ln x+\frac{10}{3}-\frac{92}{3} x+28 x^{2}, \\
& \Delta_{\mathrm{T}}^{(1)}(x)=-\frac{8}{3} x^{-1}-\left(4-8 x-16 x^{2}\right) \ln x+\frac{26}{3}+\frac{20}{3} x-4 x^{2}
\end{aligned}
$$

in the $\overline{\mathrm{MS}}$ scheme [6], see also Ref. [30]. Obviously $\Delta_{\mathrm{S}}^{(1)}$ and $\Delta_{\mathrm{T}}^{(1)}$ are much simpler than the individual NLO splitting functions. A further simplification is obtained by adding these two quantities,

$$
\Delta_{\mathrm{S}}^{(1)}(x)+\Delta_{\mathrm{T}}^{(1)}(x)=12-24 x+24 x^{2}=12 p_{\mathrm{qg}}(x),
$$

where $p_{\mathrm{qg}}(x)$ has been defined in Eq. (16). Using the results of Refs. [10-13] and Eqs. (18) and (19) the corresponding NNLO quantities are found to be (also at $x<1$ )

$$
\begin{aligned}
& \Delta_{\mathrm{S}}^{(2)}(x)=-2 \ln ^{3} x-9 \ln ^{2} x-\left(\frac{8}{3} x^{-1}+\frac{368}{9}+24 \zeta_{2}\right) \ln x+\ldots+8 \ln (1-x) \\
& \Delta_{\mathrm{T}}^{(2)}(x)=-2 \ln ^{3} x+\left(16 x^{-1}-21\right) \ln ^{2} x+\left(\frac{928}{9} x^{-1}+\frac{388}{9}+24 \zeta_{2}\right) \ln x+\ldots+8 \ln (1-x),
\end{aligned}
$$

where we have suppressed all contributions which are regular for $x \rightarrow 0$ and $x \rightarrow 1$ for brevity, and

$$
\Delta_{\mathrm{S}}^{(2)}(x)+\Delta_{\mathrm{T}}^{(2)}(x)=-24 \zeta_{2} p_{\mathrm{qg}}(x)+\text { non- } \zeta_{2} \text { terms } .
$$

The latter means the absence of $\zeta_{2}$ in the expansion about $x=1$ to all orders in $(1-x)$, cf. Ref. [31].

The absence of $\zeta_{2} x^{-1} \ln x$ also in the second line of Eq. (32) provides a check of the coefficient of $\ln x$, and hence (due to the second-moment constraint (10)) of the whole $C_{A}$ coefficient in Eq. (12). An additional $\ln x$ term in Eq. (13), except with a prefactor $C_{A}-C_{F}$, would spoil the symmetry between the two lines of Eq. (32) and hence also Eq. (33). Obviously non- $\left(C_{F}-C_{A}\right)$ terms not proportional to $p_{\mathrm{gq}}(x)$ on the r.h.s. of Eq. (13) would also conflict with the latter relation. 
A second aspect concerns the analytic structure of the physical evolution kernels, in particular the differences between the analytic continuations $(x \rightarrow 1 / x)$ of the spacelike and timelike ones. From Eq. (4) we define the matrix $\Delta K^{(n)}$ as

$$
\Delta K^{(n)}(x)=A C\left[K^{(n) S}(x)\right]-K^{(n) T}(x),
$$

with the restriction $x<1$ and entries according to Eqs. (7), (9), (12) and (13). It is interesting to investigate whether Eq. (34) directly respects Gribov-Lipatov reciprocity. In Mellin space, this feature implies that the corresponding expressions are functions only of the product $N(N+1)$ of the Mellin variable $N$, i.e., parity preserving, a fact already exploited in the large- $x$ (large- $N$ ) limit of Ref. [28]. The eigenvalues $\lambda_{i}, i=1,2$ of $\Delta K^{(n)}$ are determined from the characteristic equation

$$
\lambda_{i}^{2}-\lambda_{i} \operatorname{tr}(\Delta K)+\operatorname{det}(\Delta K)=0,
$$

and, following [29], it is sufficient to study the conditions which Gribov-Lipatov reciprocity imposes on the trace and determinant of $\Delta K^{(n)}$ in Eq. (34), i.e.,

$$
\begin{aligned}
& A C\left[\operatorname{tr}\left(\Delta K^{(n)}(x)\right)\right]-\operatorname{tr}\left(\Delta K^{(n)}(x)\right)=0, \\
& A C\left[\operatorname{det}\left(\Delta K^{(n)}(x)\right)\right]-\operatorname{det}\left(\Delta K^{(n)}(x)\right)=0 .
\end{aligned}
$$

In QCD, Eq. (36) is fulfilled to NNLO due to Eq. (9), while Eq. (37) is not. However, in the supersymmetric limit, $C_{A}=C_{F}=n_{f}$, also Eq. (37) holds to NNLO as a result of non-trivial relations between the coefficients in Eqs. (12) and (13). Eq. (37) provides thus a further constraint on $P_{\mathrm{qg}}^{(2) T}$ and $P_{\mathrm{gq}}^{(2) T}$, again except for contributions proportional to $C_{A}-C_{F}$, which vanish trivially in the transition to a $\mathcal{N}=1$ supersymmetric theory.

In summary, these considerations are still not sufficient to definitely fix the right-hand-side of Eq. (13). As an estimate of the remaining uncertainty we suggest to use the offset

$$
\delta P_{\mathrm{qg}}^{(2) T}(x)= \pm 2 \zeta_{2} \beta_{0}\left(C_{A}-C_{F}\right)(11+24 \ln x) P_{\mathrm{qg}}^{(0) T}(x) .
$$

The functions (18) and (19), the former including the error band due to Eq. (38), are shown and compared to their spacelike counterparts in Fig. 1; and the LO, NLO and NNLO approximations to Eq. (2) are illustrated in Fig. 2 at the typical scale $Q^{2} \simeq M_{Z}^{2}$. As in the diagonal cases [13], the higher-order corrections are much larger at small values of $x$ than in the spacelike case. The small- $x$ behaviour of $P_{\mathrm{qg}}^{(2) T}$ and $P_{\mathrm{gq}}^{(2) T}$ is similar to that of $P_{\mathrm{qq}}^{(2) T}$ and $P_{\mathrm{gg}}^{(2) T}$, respectively, with particularly large cancellations between the powers of $\ln x$ occurring in $P_{\mathrm{gq}}^{(2) T}$ and $P_{\mathrm{gg}}^{(2) T}$.

For the use of the NNLO splitting functions in numerical analyses we have prepared, analogous to Refs. $[10,11]$, compact and accurate parametrizations not only of the present results, but also of the non-singlet and diagonal quantities derived in Refs. [12,13]. These parametrizations can be found in Appendix B. Corresponding FORTRAN files, and FORM files of our main results, can be obtained by downloading the source of this paper from the arXiv servers or from the authors upon request. This includes the rather lengthy (even or odd) integer- $N$ Mellin-space expressions in terms of harmonic sums [33] which we have not presented here for brevity. 

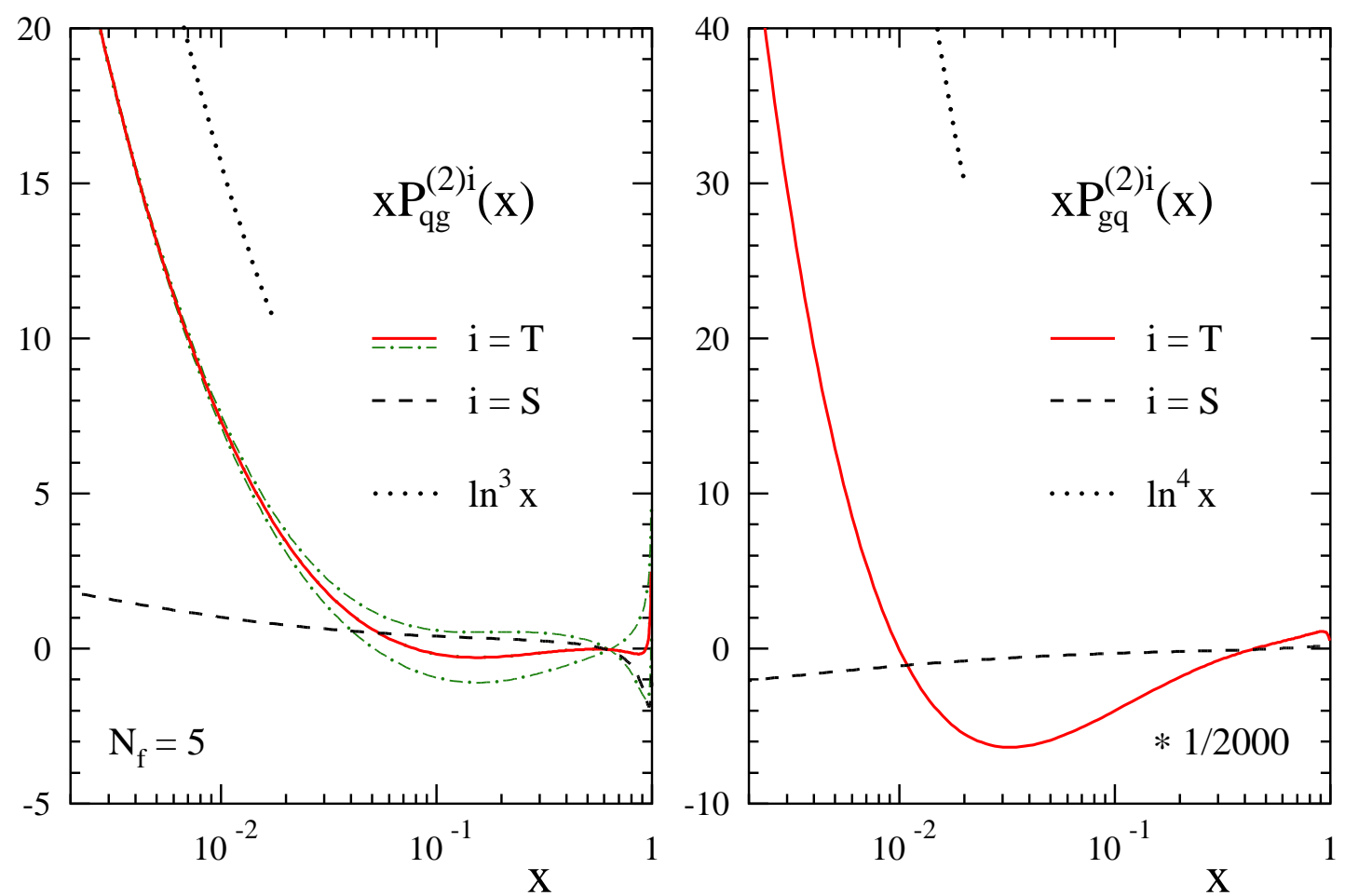

Figure 1: The third-order timelike off-diagonal (quark-gluon and gluon-quark) splitting functions for five flavours, multiplied by $x$ and divided by $2000 \simeq(4 \pi)^{3}$ for display purposes. The remaining uncertainty of the former quantity is indicated by the dash-dotted curves. Also shown are the respective leading small- $x$ contributions and the corresponding spacelike splitting functions.
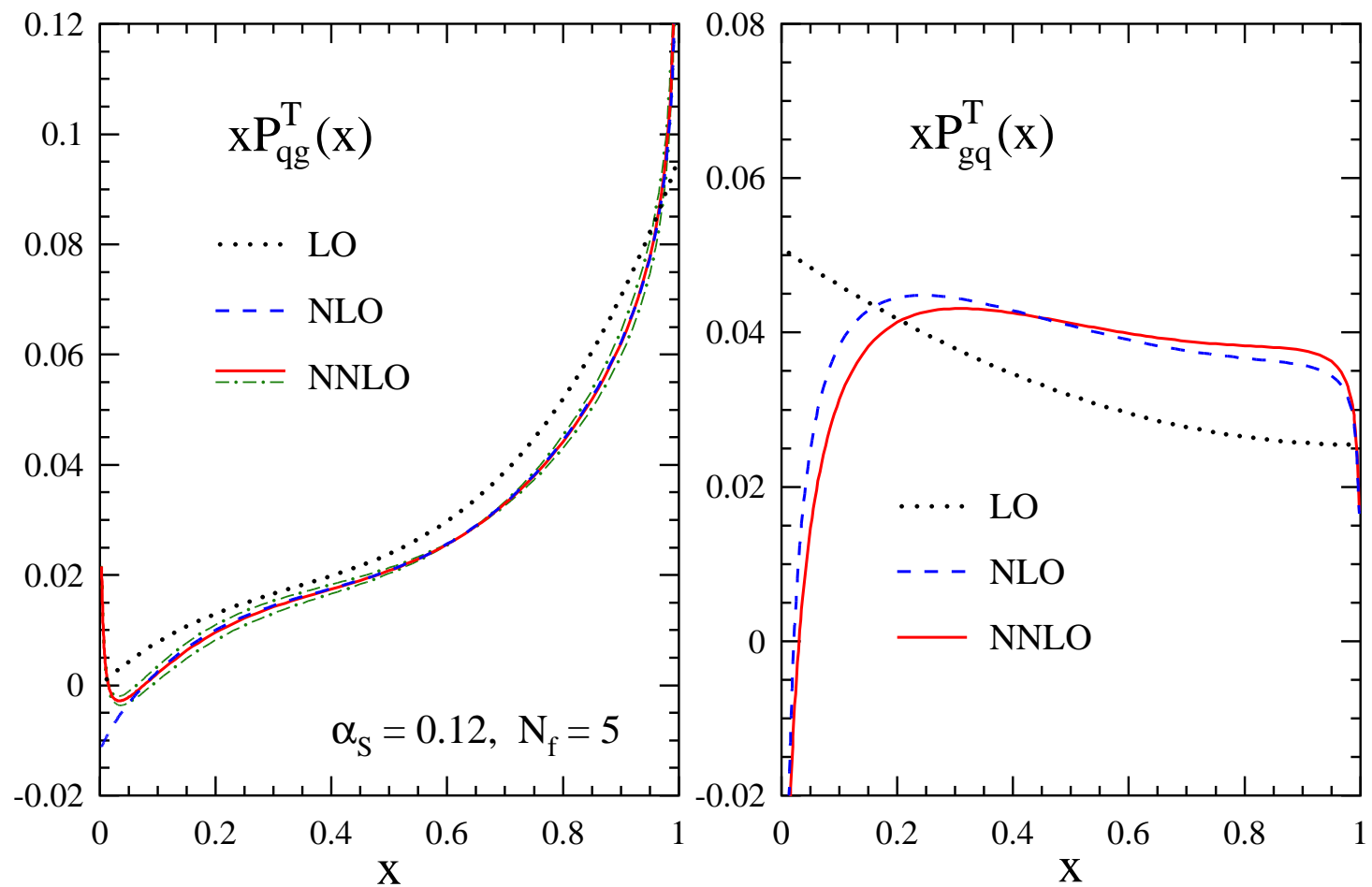

Figure 2: The resulting perturbative expansion of the timelike quark-gluon and gluon-quark splitting functions, again multiplied by $x$, at a typical value of the strong coupling constant. 
To summarize, we have performed an indirect determination of the hitherto unknown offdiagonal NNLO timelike splitting functions (18) and (19) for the evolution of parton-to-hadron fragmentation functions $D_{f}^{h}\left(x, Q^{2}\right)$. We expect the remaining uncertainty of the former quantity, estimated in Eq. (38) and illustrated in Figs. 1 and 2, to be phenomenologically acceptable. Hence, combining these results with those of Refs. [12,13,19], NNLO analyses are now possible of data on the transverse fragmentation function (but not yet its longitudinal counterpart, where also the third-order coefficient functions are required) in semi-inclusive electron-positron annihilation.

The remaining uncertainty of $P_{\mathrm{qg}}^{(2) T}$ does not affect the logarithmically enhanced large- $x$ and small- $x$ contributions. We expect that these results can be useful to improve the corresponding resummations. In fact, an extension of the generalized large- $x$ resummation of Ref. [32] to the present timelike case will be presented in a forthcoming publication.

Further research is required to completely fix $P_{\mathrm{qg}}^{(2) T}$ and to check our result for $P_{\mathrm{gq}}^{(2) T}$. A direct calculation of the leading- $n_{f}$ contribution to the former quantity should be possible, but would not address the critical contributions, while a full $x$-dependent diagram calculation beyond these terms appears to remain formidable task. A computation of the $N=4$ and $N=6$ moments of the $n_{f}^{2}$ and $n_{f}$ parts, respectively, of Eqs. (18) and (19) presumably would be sufficient and may be feasible, e.g., generalizing the approach used in Ref. [21], in the foreseeable future.

Acknowledgements: The work of A.A. and A.V has been supported by the UK Science \& Technology Facilities Council (STFC) under grant number ST/G00062X/1, and that of S.M. by the Deutsche Forschungsgemeinschaft in Sonderforschungsbereich/Transregio 9. We are also part of the European-Union funded network LHCPhenoNet, contract number PITN-GA-2010-264564. Our analytic calculations were performed using FORM [36], and the numerical evaluation of the harmonic polylogarithms was carried out using the program of Ref. [37].

\section{Appendix A}

The first- and second-order coefficient functions for SIA via an intermediate scalar in Eq. (6) read

$$
\begin{aligned}
c_{\phi, \mathrm{g}}^{(1)}(x)= & C_{A}\left(11 / 3\left(1+x+x^{2}-x_{1}^{-1}\right)+p_{\mathrm{gg}}(x)\left(8 \mathrm{H}_{0}-4 \mathrm{H}_{1}\right)+\left(67 / 9+8 \zeta_{2}\right) \delta(1-x)\right) \\
& -n_{f}\left(2 / 3\left(1+x+x^{2}-x_{1}^{-1}\right)+10 / 9 \delta(1-x)\right), \\
c_{\phi, \mathrm{q}}^{(1)}(x)= & n_{f}\left(4 x-7 x^{2}+p_{\mathrm{qg}}(x)\left(4 \mathrm{H}_{0}-2 \mathrm{H}_{1}\right)\right)
\end{aligned}
$$

with $p_{\text {gg }}(x)=x^{-1}-2+x-x^{2}+x_{1}^{-1}$, where $f(x) x_{1}^{-1} \equiv f(x) /(1-x)$ has to be read as a +-distribution if $f(x)$ does not vanish at $x=1$, and

$$
\begin{aligned}
c_{\phi, \mathrm{g}}^{(2)} & (x)=C_{A}^{2}\left(\left(5099 x^{-1}-3301 x^{2}-2570 x_{1}^{-1}\right) / 27-46 / 3+1858 / 9 x-(140 / 3+32 / 3 x\right. \\
& \left.+44 / 3\left(x^{-1}+3 x^{2}-x_{1}^{-1}\right)\right) \zeta_{2}-\left(101-2092 x^{-1}-2623 x-268\left(2 x^{2}+3 x_{1}^{-1}\right)\right) / 9 \mathrm{H}_{0} \\
& -\left(162+138 x+484 / 3 x^{2}+220 / 3\left(2 x^{-1}-x_{1}^{-1}\right)\right) \mathrm{H}_{0,0}-(268-356 x \\
& \left.-88\left(5 x^{-1}-2 x^{2}+x_{1}^{-1}\right)\right) / 3 \mathrm{H}_{2}+\left(160-46 x+340 / 3 x^{2}-778 / 9\left(x^{-1}+x_{1}^{-1}\right)\right) \mathrm{H}_{1}
\end{aligned}
$$




$$
\begin{aligned}
& -88 / 3\left(1+x+x^{2}-x_{1}^{-1}\right) \mathrm{H}_{1,0}+\left(452-308 x^{-1}-232 x+176\left(2 x^{2}-x_{1}^{-1}\right)\right) / 3 \mathrm{H}_{1,1} \\
& +8 / 3\left(6+11 x^{-1}+6 x+11 x^{2}\right) \mathrm{H}_{-1,0}-8(1+x)\left(8 \zeta_{3}+8 \zeta_{2} \mathrm{H}_{0}+22 \mathrm{H}_{0,0,0}-12 \mathrm{H}_{3}\right. \\
& \left.+4 \mathrm{H}_{2,1}\right)+4 p_{\text {gg }}(-x)\left(7 \zeta_{3}-2 \zeta_{2} \mathrm{H}_{0}+9 \mathrm{H}_{0,0,0}-2 \mathrm{H}_{3}-2 \mathrm{H}_{-2,0}-8 \zeta_{2} \mathrm{H}_{-1}+4 \mathrm{H}_{-1,2}\right. \\
& \left.-8 \mathrm{H}_{-1,-1,0}-6 \mathrm{H}_{-1,0,0}\right)+4 p_{\mathrm{gg}}(x)\left(-31 \zeta_{3}+2 \zeta_{2} \mathrm{H}_{0}+6 \mathrm{H}_{-2,0}-31 \mathrm{H}_{0,0,0}+2 \mathrm{H}_{3}\right. \\
& \left.-2 \zeta_{2} \mathrm{H}_{1}-20 \mathrm{H}_{2,0}+10 \mathrm{H}_{2,1}+6 \mathrm{H}_{1,0,0}+10 \mathrm{H}_{1,2}+10 \mathrm{H}_{1,1,0}-12 \mathrm{H}_{1,1,1}\right) \\
& \left.+\delta(1-x)\left(30425 / 162-242 / 3 \zeta_{3}+830 / 9 \zeta_{2}+101 / 5 \zeta_{2}^{2}\right)\right) \\
& +C_{A} n_{f}\left(\left(358 x^{-1}-928-502 x+672 x_{1}^{-1}\right) / 27-116 / 3 x^{2}+\left(362-260 x^{-1}-120 x_{1}^{-1}\right.\right. \\
& \left.-238 x+172 x^{2}\right) / 9 \mathrm{H}_{0}+\left(36-16 x^{-1}-12 x+40\left(x^{2}-x_{1}^{-1}\right)\right) / 3 \mathrm{H}_{0,0}-\left(350-168 x_{1}^{-1}\right. \\
& \left.-88\left(2 x^{-1}+x-3 x^{2}\right)\right) / 9 \mathrm{H}_{1}+8 / 3\left(\left(x^{-1}-1+2 x\right)\left(2 \mathrm{H}_{1,0}-\mathrm{H}_{1,1}\right)+(1+x)\left(\mathrm{H}_{2}-\zeta_{2}\right)\right) \\
& \left.-8 / 3 p_{\mathrm{gg}}(x)\left(\zeta_{2}+2 \mathrm{H}_{1,0}-4 \mathrm{H}_{1,1}+2 \mathrm{H}_{2}\right)-\delta(1-x)\left(4112 / 81+8 \zeta_{2}+28 / 3 \zeta_{3}\right)\right) \\
& +C_{F} n_{f}\left(2 x_{1}^{-1}-\left(744+723 x-2017 x^{2}+928 x^{-1}\right) / 27+\left(8+16 / 3\left(x^{-1}+x^{2}\right)+12 x\right) \zeta_{2}\right. \\
& -8 / 9\left(63+19 x^{-1}+81 x+41 x^{2}\right) \mathrm{H}_{0}+\left(14+22 x+32 / 3\left(x^{-1}+x^{2}\right)\right) \mathrm{H}_{0,0}-(12+8 x \\
& \left.+32 / 3 x^{-1}\right) \mathrm{H}_{2}+\left(78-16 x^{-1}-150 x+88 x^{2}\right) / 9 \mathrm{H}_{1}+4 / 3\left(4 x^{-1}-3 x-4 x^{2}+3\right) \mathrm{H}_{1,1} \\
& \left.+4(1+x)\left(4 \zeta_{3}+4 \zeta_{2} \mathrm{H}_{0}+11 \mathrm{H}_{0,0,0}-6 \mathrm{H}_{3}+2 \mathrm{H}_{2,1}\right)-\delta(1-x)\left(63 / 2-24 \zeta_{3}\right)\right) \\
& +n_{f}^{2}\left(8 / 27\left(1+x+x^{2}-x_{1}^{-1}\right)\left(5+3 \mathrm{H}_{1}\right)+\delta(1-x)\left(100 / 81-8 / 9 \zeta_{2}\right)\right) \text {, } \\
& c_{\phi, \mathrm{q}}^{(2)}(x)=C_{A} n_{f}\left(-\left(2590+296 x^{-1}-74 x\right) / 27-695 / 18 x^{2}+\left(22-60 x+56 x^{2}\right) \zeta_{2}\right. \\
& +(16+88 x) \zeta_{3}+\left(\left(196+48 x^{-1}-2810 x+582 x^{2}\right) / 9+16 \zeta_{2}\right) \mathrm{H}_{0}+\left(8+64 x^{-1}\right. \\
& \left.+212 x+116 x^{2}\right) / 3 \mathrm{H}_{0,0}+\left(10-32 x^{-1}-212 x+180 x^{2}\right) / 3 \mathrm{H}_{2}+(44+248 x) \mathrm{H}_{0,0,0} \\
& -8\left(1+4 x+2 x^{2}\right) \mathrm{H}_{-2,0}-\left(44+72 x+40 x^{2}\right) \mathrm{H}_{3}-8 / 3\left(3+2 x^{-1}+9 x+8 x^{2}\right) \mathrm{H}_{-1,0} \\
& -\left(10 / 3+80 x^{2}-4 / 3\left(4 x^{-1}+53 x\right)\right) \mathrm{H}_{1,1}-\left(127+24 x^{-1}-818 x+1016 x^{2}\right) / 9 \mathrm{H}_{1} \\
& -8 \mathrm{H}_{1,0}+8\left(4-2 x+6 x^{2}\right) \mathrm{H}_{2,1}+4 p_{\mathrm{qg}}(-x)\left(\zeta_{2}\left(3 \mathrm{H}_{0}-2 \mathrm{H}_{-1}\right)-5 \mathrm{H}_{-1,0,0}+2 \mathrm{H}_{-1,2}\right) \\
& \left.+p_{\mathrm{qg}}(x)\left(8 \zeta_{2} \mathrm{H}_{1}+2 / 3 \mathrm{H}_{1,0}-12 \mathrm{H}_{2,0}+16 \mathrm{H}_{1,0,0}+8 \mathrm{H}_{1,2}+8 \mathrm{H}_{1,1,0}-20 \mathrm{H}_{1,1,1}\right)\right) \\
& +C_{F} n_{f}\left(+62-71 x+155 / 2 x^{2}-\left(22-24 x+4 x^{2}\right) \zeta_{2}-\left(40-112 x+96 x^{2}\right) \zeta_{3}\right. \\
& -\left(10-47 x+98 x^{2}+16 \zeta_{2} x^{2}\right) \mathrm{H}_{0}-\left(7+80 x-104 x^{2}\right) \mathrm{H}_{0,0}+4\left(6-4 x+x^{2}\right) \mathrm{H}_{2} \\
& +16(1+x) \mathrm{H}_{-1,0}+16\left(1+2 x^{2}\right) \mathrm{H}_{-2,0}+\left(1+36 x-48 x^{2}\right) \mathrm{H}_{1}+\left(6-36 x+60 x^{2}\right) \mathrm{H}_{1,0} \\
& -4 x \mathrm{H}_{1,1}-x^{2}\left(44 \mathrm{H}_{0,0,0}+8 \mathrm{H}_{2,1}-24 \mathrm{H}_{3}\right)-8 p_{\mathrm{qg}}(-x)\left(\zeta_{2} \mathrm{H}_{-1}-\mathrm{H}_{-1,0,0}+2 \mathrm{H}_{-1,-1,0}\right) \\
& -p_{\mathrm{qg}}(x)\left(4 \zeta_{2}\left(\mathrm{H}_{0}+3 \mathrm{H}_{1}\right)+20 \mathrm{H}_{1,1}+22 \mathrm{H}_{0,0,0}+28 \mathrm{H}_{2,0}-8 \mathrm{H}_{3}+4 \mathrm{H}_{1,0,0}-12 \mathrm{H}_{1,2}\right. \\
& \left.\left.-12 \mathrm{H}_{1,1,0}+4 \mathrm{H}_{1,1,1}\right)\right) \\
& +n_{f}^{2}\left(\left(112-392 x+833 x^{2}\right) / 27-4 / 9\left(34-56 x+53 x^{2}\right) \mathrm{H}_{0}+p_{\mathrm{qg}}(x)\left(58 / 9 \mathrm{H}_{1}-4 \zeta_{2}\right.\right. \\
& \left.\left.+4 / 3\left(\mathrm{H}_{0,0}-\mathrm{H}_{2}+\mathrm{H}_{1,0}+\mathrm{H}_{1,1}\right)\right)\right)
\end{aligned}
$$

As already discussed in Ref. [13], the second moment of $c_{\phi, \mathrm{g}}^{(2)}+c_{\phi, \mathrm{q}}^{(2)}$ directly enters the NNLO Higgs decay rate to hadrons in the heavy-top limit, and agrees with the result of Ref. [34], see also Ref. [35]. We expect that these coefficient functions will be useful also for other theoretical studies. They can be employed, for instance, to extend the large- $x$ results of Ref. [28] to $P_{\mathrm{qg}}^{(2) T}$. 


\section{Appendix B}

Since the exact expressions of the NNLO splitting functions are rather lengthy and complex, it is useful to have at one's disposal compact but accurate approximate representations which also can be transformed readily to Mellin space at all (complex) values of $N$. In this final appendix we therefore provide parametrizations of all NNLO timelike splitting functions in QCD which are built up, besides powers of $x$, only from the +-distribution and the end-point logarithms

$$
\mathcal{D}_{0}=1 /(1-x)_{+}, \quad L_{1}=\ln (1-x), \quad L_{0}=\ln x .
$$

The non-singlet splitting functions $P_{\mathrm{ns}}^{(2) T \pm}[12]$ can be represented by

$$
\begin{aligned}
& P_{\mathrm{ns}}^{(2) T+}(x) \cong+ 1174.898 \mathcal{D}_{0}+1295.625 \delta(1-x)-707.67 L_{1}+1658.7-4249.4 x \\
&- 1075.3 x^{2}+593.9 x^{3}-L_{0} L_{1}\left[56.907+519.37 L_{0}+559.1 x\right] \\
&+ 1327.5 L_{0}-189.37 L_{0}^{2}-352 / 9 L_{0}^{3}+128 / 81 L_{0}^{4} \\
&+\quad n_{f}\left(-183.187 \mathcal{D}_{0}-173.935 \delta(1-x)+5120 / 81 L_{1}-198.1+466.29 x\right. \\
& \quad+181.18 x^{2}-31.84 x^{3}-39.113 x L_{0}-L_{0} L_{1}[50.758-85.72 x \\
&\left.\left.\quad-28.551 L_{0}+23.102 x L_{0}\right]-168.89 L_{0}-176 / 81 L_{0}^{2}+64 / 27 L_{0}^{3}\right) \\
&+n_{f}^{2}\left(-\mathcal{D}_{0}-\left(51 / 16+3 \zeta_{3}-5 \zeta_{2}\right) \delta(1-x)+x(1-x)^{-1} L_{0}\left(3 / 2 L_{0}+5\right)\right. \\
&\left.\quad+1+(1-x)\left(6+11 / 2 L_{0}+3 / 4 L_{0}^{2}\right)\right) 64 / 81
\end{aligned}
$$

and

$$
\begin{aligned}
P_{\mathrm{ns}}^{(2) T-}(x) \cong & +1174.898 \mathcal{D}_{0}+1295.622 \delta(1-x)-707.94 L_{1}+1981.3-4885.7 x \\
& -577.42 x^{2}+407.89 x^{3}+L_{0} L_{1}\left[4563.2+1905.4 L_{0}-5140.6 x\right. \\
+ & \left.1969.5 x L_{0}\right]-34.683 x L_{0}^{4}-437.03 x L_{0}^{3}+1625.5 L_{0}-38.298 L_{0}^{2} \\
& -1024 / 27 L_{0}^{3}-140 / 81 L_{0}^{4} \\
+\quad n_{f} & \left(-183.187 \mathcal{D}_{0}-173.9376 \delta(1-x)+5120 / 81 L_{1}-217.84+511.92 x\right. \\
& +209.19 x^{2}-85.786 x^{3}+92.453 x L_{0}+L_{0} L_{1}\left[71.428+30.554 L_{0}\right. \\
& \left.\left.-23.722 x-18.975 x L_{0}\right]-188.99 L_{0}-784 / 81 L_{0}^{2}+128 / 81 L_{0}^{3}\right) \\
+\quad n_{f}^{2} & \left(-\mathcal{D}_{0}-\left(51 / 16+3 \zeta_{3}-5 \zeta_{2}\right) \delta(1-x)+x(1-x)^{-1} L_{0}\left(3 / 2 L_{0}+5\right)\right. \\
& \left.+1+(1-x)\left(6+11 / 2 L_{0}+3 / 4 L_{0}^{2}\right)\right) 64 / 81 .
\end{aligned}
$$

The $n_{f}^{2}$ parts of $P_{\mathrm{ns}}^{(2) T \pm}$ (which are identical and equal to their spacelike counterparts in Ref. [10]), the +-distribution contributions (up to a numerical truncation of the coefficients involving $\zeta_{i}$ ), and the rational coefficients of the (sub-)leading regular end-point terms are exact in Eqs. (B.1) and (B.2). The remaining coefficients have been determined by fits to the exact results at $10^{-6} \leq x \leq$ $1-10^{-6}$, and finally the coefficients of $\delta(1-x)$ have been adjusted very slightly using the lowest integer moments. The difference between the NNLO 'valence' and 'minus' splitting functions is equal to that in the spacelike case; a parametrization can be found in Eq. (4.24) of Ref. [10]). 
Corresponding representations of the pure-singlet and gluon-gluon splitting functions [13] are

$$
\begin{aligned}
P_{\mathrm{ps}}^{(2) T}(x) \cong\left\{n_{f}\right. & \left(-5.926 L_{1}^{3}-9.751 L_{1}^{2}-8.65 L_{1}-106.65-848.97 x+368.79 x^{2}\right. \\
& -61.284 x^{3}+96.171 L_{0} L_{1}+656.49 L_{0}+425.14 L_{0}^{2}+47.322 L_{0}^{3} \\
& \left.+9.072 L_{0}^{4}+479.87 x^{-1}+324.07 x^{-1} L_{0}-128 / 9 x^{-1} L_{0}^{2}-256 / 9 x^{-1} L_{0}^{3}\right) \\
+\quad n_{f}^{2} & \left(1.778 L_{1}^{2}+16.611 L_{1}+87.795-57.688 x-41.827 x^{2}+25.628 x^{3}\right. \\
& -7.9934 x^{4}-2.1031 L_{0} L_{1}+26.294 x L_{0}-7.8645 x L_{0}^{3}+57.713 L_{0} \\
& \left.\left.+9.1682 L_{0}^{2}-1.9 L_{0}^{3}+0.019122 L_{0}^{4}-128 / 81 x^{-1}\right)\right\}(1-x)
\end{aligned}
$$

and

$$
\begin{aligned}
P_{\mathrm{gg}}^{(2) T}(x) \cong+ & 2643.521 \mathcal{D}_{0}+4425.451 \delta(1-x)-3590.1 L_{1}-28489+7469 x \\
+ & 30421 x^{2}-53017 x^{3}+19556 x^{4}-L_{0} L_{1}\left(186.4+21328 L_{0}\right)+12258 L_{0} \\
+ & 13528 L_{0}^{2}+3281.7 L_{0}^{3}+191.99 L_{0}^{4}+5685.8 x L_{0}^{3}+14214.4 x^{-1} \\
+ & 10233 x^{-1} L_{0}+3651.1 x^{-1} L_{0}^{2}+3168 x^{-1} L_{0}^{3}+576 x^{-1} L_{0}^{4} \\
+\quad n_{f} & \left(-412.172 \mathcal{D}_{0}-528.719 \delta(1-x)+319.97 L_{1}+248.95+260.6 x\right. \\
& +272.79 x^{2}+2133.2 x^{3}-926.87 x^{4}+L_{0} L_{1}\left(1266.5-29.709 L_{0}\right. \\
& \left.+87.771 L_{1}\right)+4.9934 L_{0}+482.94 L_{0}^{2}+155.1 L_{0}^{3}+18.085 L_{0}^{4} \\
& \left.+485.18 x L_{0}^{3}-804.13 x^{-1}-5.47 x^{-1} L_{0}+2368 / 9 x^{-1} L_{0}^{2}+448 / 9 x^{-1} L_{0}^{3}\right) \\
+\quad n_{f}^{2} & \left(-16 / 9 \mathcal{D}_{0}+6.4628 \delta(1-x)-77.19+153.27 x-106.03 x^{2}+11.995 x^{3}\right. \\
& -L_{0} L_{1}\left(115.01-96.522 x+62.908 L_{0}\right)-69.712 L_{0}-44.8 L_{0}^{2}-5.037 L_{0}^{3} \\
& \left.+472 / 243 x^{-1}+368 / 81 x^{-1} L_{0}+32 / 27 x^{-1} L_{0}^{2}\right) .
\end{aligned}
$$

In Eqs. (B.3) and (B.4) the small- $x$ leading terms are exact up to truncations of irrational numbers. The same holds for the coefficients of $L_{1}, L_{1}^{2}$ and $L_{1}^{3}$ in Eq. (B.3) and that of $\mathcal{D}_{0}$ in Eq. (B.4), where the coefficient of $\delta(1-x)$ has been minimally adjusted using the lowest moments.

The new NNLO off-diagonal quantities (18) and (19) can finally be parametrized as

$$
\begin{aligned}
P_{\mathrm{qg}}^{(2) T}(x) \cong n_{f} & \left(100 / 27 L_{1}^{4}+350 / 9 L_{1}^{3}+263.07 L_{1}^{2}+693.84 L_{1}+603.71-882.48 x\right. \\
& +4723.2 x^{2}-4745.8 x^{3}-175.28 x^{4}-L_{0} L_{1}(1809.4+107.59 x) \\
& -885.5 x L_{0}^{4}+1864 L_{0}+1512 L_{0}^{2}+361.28 L_{0}^{3}+42.328 L_{0}^{4}+1141.7 x^{-1} \\
& \left.+675.83 x^{-1} L_{0}-64 x^{-1}\left(L_{0}^{2}+L_{0}^{3}\right)\right) \\
+n_{f}^{2}( & -100 / 27 L_{1}^{3}-35.446 L_{1}^{2}-103.609 L_{1}-113.81+341.26 x-853.35 x^{2} \\
& +492.1 x^{3}+14.803 x^{4}+L_{0} L_{1}\left(966.96-1.593 L_{1}-709.1 x\right)-333.8 x L_{0}^{3} \\
& +619.75 L_{0}+255.62 L_{0}^{2}+21.569 L_{0}^{3}-2.8986 x^{-1}-3.1752 x^{-1} L_{0} \\
& \left.-32 / 27 x^{-1} L_{0}^{2}\right) \\
+ & n_{f}^{3}
\end{aligned}
$$

where the $n_{f}^{3}$ part is exact, and 


$$
\begin{aligned}
P_{\mathrm{gq}}^{(2) T}(x) \cong \quad & 400 / 81 L_{1}^{4}+520 / 27 L_{1}^{3}-220.13 L_{1}^{2}-152.6 L_{1}+272.85-7188.7 x \\
& +5693.2 x^{2}+146.98 x^{3}+128.19 x^{4}-L_{0} L_{1}\left(1300.6+71.23 L_{1}\right)+543.8 x L_{0}^{3} \\
+ & 4.4136 L_{0}-0.71252 L_{0}^{2}-126.38 L_{0}^{3}-30.061 L_{0}^{4}+5803.7 x^{-1} \\
+ & 4776.5 x^{-1} L_{0}+1001.89 x^{-1} L_{0}^{2}+3712 / 3 x^{-1} L_{0}^{3}+256 x^{-1} L_{0}^{4} \\
+\quad n_{f} & \left(80 / 81 L_{1}^{3}+1040 / 81 L_{1}^{2}-16.914 L_{1}-871.3+790.13 x-241.23 x^{2}\right. \\
& +43.252 x^{3}-4.3465 x L_{0}^{3}+55.048 L_{0} L_{1}-492 L_{0}-343.1 L_{0}^{2}-48.6 L_{0}^{3} \\
& \left.+6.0041 x^{-1}+141.93 x^{-1} L_{0}+2912 / 27 x^{-1} L_{0}^{2}+1280 / 81 x^{-1} L_{0}^{3}\right) .
\end{aligned}
$$

The coefficients of the leading small- $x$ terms are exact in Eqs. (B.5) and (B.6), up to a truncation of irrational numbers. The same holds for the coefficients of $L_{0}^{2}, L_{0}^{3}$ and $L_{0}^{4}$ in Eq. (B.6). The coefficients of the large- $x$ terms are also partially exact.

Except for values of $x$ very close to zeros of the splitting functions, the parametrizations. (B.1) (B.6) deviate from the exact expressions by less than one part in a thousand, which should be amply sufficient for foreseeable numerical applications. Also the complex- $N$ moments of the splitting functions can be readily obtained to a perfectly sufficient accuracy using the above representations. The Mellin transform of Eqs. (B.1) - (B.6) involve only simple harmonic sums (see, e.g, the appendix of Ref. [38]) of which the analytic continuations in terms of logarithmic derivatives of Euler's $\Gamma$-function are well known.

\section{References}

[1] K. Nakamura et al. [Particle Data Group], J. Phys. G37 (2010) 075021

[2] H. Georgi and H.D. Politzer, Nucl. Phys. B136 (1978) 445

[3] G. Altarelli and G. Parisi, Nucl. Phys. B126 (1977) 298

[4] V.N. Gribov and L.N. Lipatov, Sov. J. Nucl. Phys. 15 (1972) 438, ibid. 675

[5] G. Curci, W. Furmanski and R. Petronzio, Nucl. Phys. B175 (1980) 27

[6] W. Furmanski and R. Petronzio, Phys. Lett. 97B (1980) 437

[7] J. Kalinowski, K. Konishi, P.N. Scharbach and T. R. Taylor, Nucl. Phys. B181 (1981) 221,

J. Kalinowski, K. Konishi and T. R. Taylor, Nucl. Phys. B181 (1981) 253

[8] E.G. Floratos, C. Kounnas and R. Lacaze, Nucl. Phys. B192 (1981) 417

[9] T. Munehisa, H. Okada, K. Kudoh and K. Kitani, Prog. Theor. Phys. 67 (1982) 609

[10] S. Moch, J.A.M. Vermaseren and A. Vogt, Nucl. Phys. B688 (2004) 101, hep-ph/0403192

[11] A. Vogt, S. Moch and J.A.M. Vermaseren, Nucl. Phys. B691 (2004) 129, hep-ph/0404111

[12] A. Mitov, S. Moch and A. Vogt, Phys. Lett. B638 (2006) 61, hep-ph/0604053

[13] S. Moch and A. Vogt, Phys. Lett. B659 (2008) 290, arXiv:0709.3899

[14] Y.L. Dokshitzer, G. Marchesini and G.P. Salam, Phys. Lett. B634 (2006) 504, hep-ph/0511302 
[15] J. Blümlein, V. Ravindran, W.L. van Neerven, Nucl. Phys. B586 (2000) 349, hep-ph/0004172

[16] M. Stratmann and W. Vogelsang, Nucl. Phys. B496 (1997) 41, hep-ph/9612250

[17] G. Soar, S. Moch, J.A.M. Vermaseren and A. Vogt, Nucl. Phys. B832 (2010) 152, arXiv:0912.0369

[18] E.B. Zijlstra and W.L. van Neerven, Phys. Lett. B272 (1991) 127, Phys. Lett. B273 (1991) 476

[19] P.J. Rijken and W.L. van Neerven, Nucl. Phys. B488 (1997) 233, hep-ph/9609377

[20] S. Moch and J.A.M. Vermaseren, Nucl. Phys. B573 (2000) 853, hep-ph/9912355

[21] A. Mitov and S. Moch, Nucl. Phys. B751 (2006) 18, hep-ph/0604160

[22] A. Daleo, A. Gehrmann-De Ridder, T. Gehrmann, G. Luisoni, JHEP 01 (2010) 118, arXiv:0912.0374

[23] D.J. Broadhurst and A.L. Kataev, Phys. Lette B315 (1993) 179

[24] R.J. Crewther, Phys. Lett. B397 (1997) 137, hep-ph/9701321, P.A. Baikov, K.G. Chetyrkin and J.H. Kühn, Phys. Rev. Lett. 104 (2010) 132004, arXiv:1001.3606

[25] E. Remiddi and J.A.M. Vermaseren, Int. J. Mod. Phys. A15 (2000) 725, hep-ph/9905237

[26] A.H. Mueller, Phys. Lett. B104 (1981) 161;

A. Bassetto, M. Ciafaloni, G. Marchesini and A.H. Mueller, Nucl. Phys. B207 (1982) 189

[27] A. Vogt, Phys. Lett. B691 (2010) 77, arXiv:1005.1606

[28] G. Grunberg, arXiv:1101.5377

[29] B. Basso and G.P. Korchemsky, Nucl. Phys. B775 (2007) 1, hep-th/0612247

[30] I. Antoniadis and E.G. Floratos, Nucl. Phys. B191 (1981) 217

[31] S. Moch and A. Vogt, JHEP 11 (2009) 099, arXiv:0909.2124

[32] A.A. Almasy, G. Soar and A. Vogt, JHEP 03 (2011) 030, arXiv:1012.3352

[33] J.A.M. Vermaseren, Int. J. Mod. Phys. A14 (1999) 2037, hep-ph/9806280

[34] K.G. Chetyrkin, B.A. Kniehl and M. Steinhauser, Phys. Rev. Lett. 79 (1997) 353, hep-ph/9705240

[35] M. Schreck and M. Steinhauser, Phys. Lett. B655 (2007) 148, arXiv:0708.0916

[36] J.A.M. Vermaseren, New features of FORM, math-ph/0010025

[37] T. Gehrmann and E. Remiddi, Comput. Phys. Commun. 141 (2001) 296, hep-ph/0107173

[38] J. Blümlein and S. Kurth, Phys. Rev. D60 (1999) 014018, hep-ph/9810241 